Document downloaded from:

http://hdl.handle.net/10251/169903

This paper must be cited as:

Bernardes, NCJ.; Bonilla, A.; Peris Manguillot, A. (2020). Mean Li-Yorke chaos in Banach spaces. Journal of Functional Analysis. 278(3):1-31.

https://doi.org/10.1016/j.jfa.2019.108343

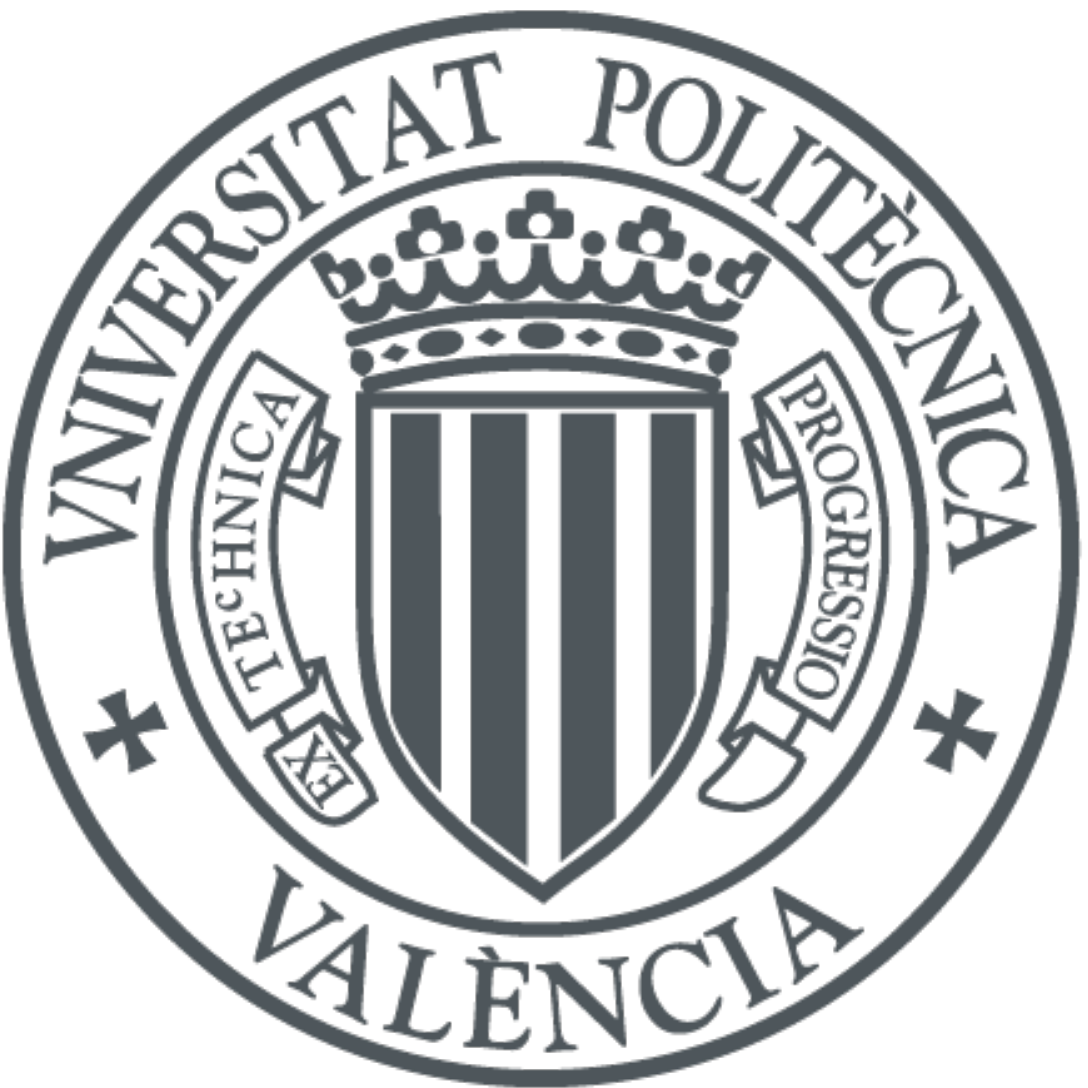

The final publication is available at

https://doi.org/10.1016/j.jfa.2019.108343

Copyright Elsevier

Additional Information 


\title{
Mean Li-Yorke chaos in Banach spaces
}

\author{
N. C. Bernardes Jr., A. Bonilla and A. Peris
}

\begin{abstract}
We investigate the notion of mean Li-Yorke chaos for operators on Banach spaces. We show that it differs from the notion of distributional chaos of type 2, contrary to what happens in the context of topological dynamics on compact metric spaces. We prove that an operator is mean Li-Yorke chaotic if and only if it has an absolutely mean irregular vector. As a consequence, absolutely Cesàro bounded operators are never mean Li-Yorke chaotic. Dense mean Li-Yorke chaos is shown to be equivalent to the existence of a dense (or residual) set of absolutely mean irregular vectors. As a consequence, every mean Li-Yorke chaotic operator is densely mean Li-Yorke chaotic on some infinite-dimensional closed invariant subspace. A (Dense) Mean Li-Yorke Chaos Criterion and a sufficient condition for the existence of a dense absolutely mean irregular manifold are also obtained. Moreover, we construct an example of an invertible hypercyclic operator $T$ such that every nonzero vector is absolutely mean irregular for both $T$ and $T^{-1}$. Several other examples are also presented. Finally, mean Li-Yorke chaos is also investigated for $C_{0}$-semigroups of operators on Banach spaces. ${ }^{1}$
\end{abstract}

\section{Introduction}

In recent years, it has become popular in the area of dynamical systems to investigate notions related to averages involving orbits or pseudo-orbits, such as mean Li-Yorke chaos [24, 29], mean equicontinuity and mean sensitivity [31], and notions of shadowing with average error in tracing [39].

Our goal in this work is to investigate the notion of mean Li-Yorke chaos for operators on Banach spaces. It turns out that this notion is intimately related to the notion of absolutely mean irregular vector. Moreover, we also establish some results on absolutely Cesàro bounded operators.

Let us now present the relevant definitions for our work.

Definition 1. An operator $T$ on a Banach space $X$ is said to be mean Li-Yorke chaotic if there is an uncountable subset $S$ of $X$ (a mean L $i$-Yorke set for $T$ ) such that every pair $(x, y)$ of distinct points in $S$ is a mean Li-Yorke pair for $T$, in the sense that

$$
\liminf _{N \rightarrow \infty} \frac{1}{N} \sum_{j=1}^{N}\left\|T^{j} x-T^{j} y\right\|=0 \quad \text { and } \quad \limsup _{N \rightarrow \infty} \frac{1}{N} \sum_{j=1}^{N}\left\|T^{j} x-T^{j} y\right\|>0 .
$$

If $S$ can be chosen to be dense (resp. residual) in $X$, then we say that $T$ is densely (resp. generically) mean Li-Yorke chaotic.

\footnotetext{
${ }^{1} 2010$ Mathematics Subject Classification: 47A16, 37D45.

Keywords: Banach spaces, operators, mean Li-Yorke chaos, absolute Cesàro boundedness, absolutely mean irregular vector, distributional chaos, hypercyclicity.
} 
Definition 2. Given an operator $T$ and a vector $x$, we say that $x$ is an absolutely mean irregular (resp. absolutely mean semi-irregular) vector for $T$ if

$$
\liminf _{N \rightarrow \infty} \frac{1}{N} \sum_{j=1}^{N}\left\|T^{j} x\right\|=0 \quad \text { and } \quad \limsup _{N \rightarrow \infty} \frac{1}{N} \sum_{j=1}^{N}\left\|T^{j} x\right\|=\infty(\text { resp. }>0) .
$$

Definition 3. [28] An operator $T$ is said to be absolutely Cesàro bounded if there exists a constant $C>0$ such that

$$
\sup _{N \in \mathbb{N}} \frac{1}{N} \sum_{j=1}^{N}\left\|T^{j} x\right\| \leq C\|x\| \quad \text { for all } x \in X
$$

Also related to our work is the notion of distributional chaos. Actually, there are at least four different notions of distributional chaos, namely DC1, DC2, DC2 $\frac{1}{2}$ and DC3 (see Section 2 for the definitions). For (nonlinear) dynamical systems on compact metric spaces, mean Li-Yorke chaos is equivalent to DC2 [20], which is not equivalent to DC1 [36]. The situation is different in our context. Indeed, we saw in [13] that DC1 and DC2 are always equivalent for operators on Banach spaces, but we will show that DC2 is not equivalent to mean Li-Yorke chaos in this context. The paper is organized as follows.

Section 2 recalls some definitions and fixes the notation.

In Section 3 we prove that an operator is mean Li-Yorke chaotic if and only if it has an absolutely mean irregular vector. As a consequence, no absolutely Cesàro bounded operator is mean Li-Yorke chaotic. We also establish a Mean Li-Yorke Chaos Criterion and present several examples, including an example of a DC1 (= DC2) operator which is not mean Li-Yorke chaotic. Finally, we show that the Frequent Hypercyclicity Criterion implies mean Li-Yorke chaos.

Section 4 is devoted to show that dense mean Li-Yorke chaos is equivalent to the existence of a dense (or residual) set of absolutely mean irregular vectors. As a consequence, every mean Li-Yorke chaotic operator is densely mean Li-Yorke chaotic on some infinite-dimensional closed invariant subspace. We also establish a Dense Mean Li-Yorke Criterion and several sufficient conditions for dense mean Li-Yorke chaos. As an application, we give an example of a densely mean Li-Yorke chaotic operator which is not Cesàro Hypercyclic.

In Section 5 we establish a sufficient condition for the existence of a dense absolutely mean irregular manifold. As an application, we obtain a dichotomy for unilateral weighted backward shifts $B_{w}$, which says that either $\frac{1}{N} \sum_{j=1}^{N}\left\|\left(B_{w}\right)^{j} x\right\| \rightarrow 0$ for all $x \in X$ or $B_{w}$ admits a dense absolutely mean irregular manifold. By using this dichotomy, we give an example of a densely mean Li-Yorke chaotic operator which is not hypercyclic.

In Section 6 we give some characterizations for generic mean Li-Yorke chaos and construct an example of an invertible hypercyclic operator $T$ such that both $T$ and $T^{-1}$ are completely absolutely mean irregular.

Finally, Section 7 is devoted to the study of mean Li-Yorke chaos for $C_{0}$-semigroups of operators on Banach spaces.

\section{Notations and preliminaries}

Throughout this paper $X$ will denote an arbitrary Banach space, unless otherwise specified, and $L(X)$ is the space of all bounded linear operators on $X$. 
Let us recall that $T \in L(X)$ is said to be $L i$-Yorke chaotic if there exists an uncountable set $\Gamma \subset X$ such that for every pair $(x, y) \in \Gamma \times \Gamma$ of distinct points, we have

$$
\liminf _{n \rightarrow \infty}\left\|T^{n} x-T^{n} y\right\|=0 \text { and } \quad \limsup _{n \rightarrow \infty}\left\|T^{n} x-T^{n} y\right\|>0 .
$$

$\Gamma$ is called a scrambled set for $T$ and each such pair $(x, y)$ is called a $L i$-Yorke pair for $T$. We easily observe that mean Li-Yorke chaos implies Li-Yorke chaos since the corresponding conditions in Definition 1 expressed in terms of limits (inf and sup) of means obviously imply the ones of Li-Yorke chaos without means.

We also recall that the lower and the upper densities of a set $A \subset \mathbb{N}$ are defined as

$$
\underline{\operatorname{dens}}(A):=\liminf _{n \rightarrow \infty} \frac{\operatorname{card}(A \cap[1, n])}{n} \text { and } \overline{\operatorname{dens}}(A):=\limsup _{n \rightarrow \infty} \frac{\operatorname{card}(A \cap[1, n])}{n},
$$

respectively. Given $T \in L(X), x, y \in X$ and $\delta>0$, the lower and the upper distributional functions of $x, y$ associated to $T$ are defined by

$$
\begin{aligned}
& F_{x, y}(\delta):=\underline{\operatorname{dens}}\left(\left\{j \in \mathbb{N}:\left\|T^{j} x-T^{j} y\right\|<\delta\right\}\right), \\
& F_{x, y}^{*}(\delta):=\overline{\operatorname{dens}}\left(\left\{j \in \mathbb{N}:\left\|T^{j} x-T^{j} y\right\|<\delta\right\}\right),
\end{aligned}
$$

respectively. If the pair $(x, y)$ satisfies

(DC1) $F_{x, y}^{*} \equiv 1$ and $F_{x, y}(\varepsilon)=0$ for some $\varepsilon>0$, or

(DC2) $F_{x, y}^{*} \equiv 1$ and $F_{x, y}(\varepsilon)<1$ for some $\varepsilon>0$, or

(DC2 $\left.\frac{1}{2}\right)$ There exist $c>0$ and $r>0$ such that $F_{x, y}(\delta)<c<F_{x, y}^{*}(\delta)$ for all $0<\delta<r$, or

(DC3) $F_{x, y}(\delta)<F_{x, y}^{*}(\delta)$ for all $\delta$ in a nondegenerate interval $J \subset(0, \infty)$,

then $(x, y)$ is called a distributionally chaotic pair of type $k \in\left\{1,2,2 \frac{1}{2}, 3\right\}$ for $T$. The operator $T$ is said to be distributionally chaotic of type $k$ (DCk) if there exists an uncountable set $\Gamma \subset X$ such that every pair $(x, y)$ of distinct points in $\Gamma$ is a distributionally chaotic pair of type $k$ for $T$. In this case, $\Gamma$ is a distributionally scrambled set of type $k$ for $T$. Distributional chaos of type 1 is often called simply distributional chaos.

For operators on Banach spaces, DC1 and DC2 are always equivalent [13, Theorem 2], and imply Li-Yorke chaos. Li-Yorke chaos and distributional chaos for linear operators have been studied in $[6,8,10,11,12,13,14,27,28,32,33,38,40,41,42]$, for instance.

Let us also recall that $T \in L(X)$ is frequently hypercyclic $(\mathrm{FH})$, upper-frequently hypercyclic $(\mathrm{UFH})$, reiteratively hypercyclic $(\mathrm{RH})$ or hypercyclic $(\mathrm{H})$ if there exists $x \in X$ such that for every nonempty open subset $U$ of $X$, the set $\left\{n \in \mathbb{N}: T^{n} x \in U\right\}$ has positive lower density, has positive upper density, has positive upper Banach density or is nonempty, respectively. $T$ is Cesàro hypercyclic if there exists $x \in X$ such that the sequence $\left(\frac{1}{n} \sum_{j=0}^{n-1} T^{j} x\right)_{n \in \mathbb{N}}$ is dense in $X$. Moreover, $T$ is mixing if for every nonempty open sets $U, V \subset X$, there exists $n_{0} \in \mathbb{N}$ such that $T^{n}(U) \cap V \neq \emptyset$ for all $n \geq n_{0}$, $T$ is weakly-mixing if $T \oplus T$ is hypercyclic, and $T$ is Devaney chaotic if it is hypercyclic and has a dense set of periodic points. See $[4,5,15,16,25,26]$, for instance.

Finally, the orbit of $x$ is distributionally near to 0 if there is $A \subset \mathbb{N}$ with $\overline{\operatorname{dens}}(A)=1$ such that $\lim _{n \in A} T^{n} x=0$. We say that $x$ has a distributionally unbounded orbit if there is $B \subset \mathbb{N}$ with $\overline{\operatorname{dens}}(B)=1$ such that $\lim _{n \in B}\left\|T^{n} x\right\|=\infty$. If the orbit of $x$ has both properties, then $x$ is a distributionally irregular vector for $T$. It was proved in [11] that $T$ is distributionally chaotic if and only if $T$ has a distributionally irregular vector. 


\section{Mean Li-Yorke chaotic operators and absolutely mean irregular vectors}

We begin with some useful characterizations of absolutely Cesàro bounded operators.

Theorem 4. For every $T \in L(X)$, the following assertions are equivalent:

(i) $T$ is not absolutely Cesàro bounded;

(ii) There is a vector $x \in X$ such that

$$
\sup _{N \in \mathbb{N}} \frac{1}{N} \sum_{j=1}^{N}\left\|T^{j} x\right\|=\infty
$$

(iii) The set of all vectors $y \in X$ such that

$$
\sup _{N \in \mathbb{N}} \frac{1}{N} \sum_{j=1}^{N}\left\|T^{j} y\right\|=\infty
$$

is residual in $X$.

Proof. The implications (iii) $\Rightarrow$ (ii) $\Rightarrow$ (i) are trivial.

(i) $\Rightarrow$ (ii): Since $T$ is not absolutely Cesàro bounded, given $\delta>0$ and $C>0$, there exist $x \in X$ and $N \in \mathbb{N}$ so that

$$
\|x\|<\delta \quad \text { and } \quad \frac{1}{N} \sum_{j=1}^{N}\left\|T^{j} x\right\|>C .
$$

Then, we can define inductively sequences $\left(x_{n}\right)$ in $X$ and $\left(N_{n}\right)$ increasing in $\mathbb{N}$ so that $\left\|x_{n}\right\|<2^{-n}$ for all $n$, and

$$
\frac{1}{N_{k}} \sum_{j=1}^{N_{k}}\left\|T^{j}\left(x_{1}+\cdots+x_{n}\right)\right\|>k \quad \text { whenever } 1 \leq k \leq n .
$$

Indeed, let $x_{1}, \ldots, x_{n-1}$ in $X$ and $\left(N_{k}\right)_{k=1}^{n-1}$ be chosen so that

$$
\left\|x_{k}\right\|<2^{-k} \text { and } \frac{1}{N_{k}} \sum_{j=1}^{N_{k}}\left\|T^{j}\left(x_{1}+\cdots+x_{n-1}\right)\right\|>k \quad \text { for } 1 \leq k \leq n-1 .
$$

We select $y_{n} \in X$ and $N_{n}>N_{n-1}$ with $\left\|y_{n}\right\|<2^{-n}$,

$\frac{1}{N_{k}} \sum_{j=1}^{N_{k}}\left\|T^{j}\left(x_{1}+\cdots+x_{n-1}+y_{n}\right)\right\|>k$ for $1 \leq k \leq n-1$, and $\frac{1}{N_{n}} \sum_{j=1}^{N_{n}}\left\|T^{j}\left(y_{n}\right)\right\|>2 n$.

Now, two cases may happen: If $N_{n}^{-1} \sum_{j=1}^{N_{n}}\left\|T^{j}\left(x_{1}+\cdots+x_{n-1}\right)\right\|>n$, we select $x_{n}=0$. Otherwise, $x_{n}=y_{n}$, and we are done. 
Let $x:=\sum_{n=1}^{\infty} x_{n} \in X$. Then

$$
\frac{1}{N_{k}} \sum_{j=1}^{N_{k}}\left\|T^{j} x\right\| \geq k \quad \text { for all } k \in \mathbb{N}
$$

which gives (ii).

(ii) $\Rightarrow$ (iii): Let $A$ denote the set considered in (iii). Since

$$
A=\bigcap_{k=1}^{\infty} \bigcup_{N=1}^{\infty}\left\{y \in X: \frac{1}{N} \sum_{j=1}^{N}\left\|T^{j} y\right\|>k\right\}
$$

we have that $A$ is a $G_{\delta}$ set. If $z \in X \backslash A$ then

$$
\sup _{N \in \mathbb{N}} \frac{1}{N} \sum_{j=1}^{N}\left\|T^{j} z\right\|<\infty .
$$

Let $x \in X$ be a vector given by (ii). Then

$$
\sup _{N \in \mathbb{N}} \frac{1}{N} \sum_{j=1}^{N}\left\|T^{j}(z+\lambda x)\right\|=\infty \quad \text { whenever } \lambda \neq 0,
$$

which implies that $z \in \bar{A}$. Thus, $A$ is dense in $X$.

Let us now prove that mean Li-Yorke chaos is equivalent to the existence of an absolutely mean irregular vector.

Theorem 5. For every $T \in L(X)$, the following assertions are equivalent:

(i) $T$ is mean Li-Yorke chaotic;

(ii) T has a mean Li-Yorke pair;

(iii) $T$ has an absolutely mean semi-irregular vector;

(iv) T has an absolutely mean irregular vector;

(v) The restriction of $T$ to some infinite-dimensional closed $T$-invariant subspace $Y$ has a residual set of absolutely mean irregular vectors.

Proof. The implications (v) $\Rightarrow$ (iv) $\Rightarrow$ (iii) are trivial.

(iii) $\Rightarrow$ (ii): If $x$ is an absolutely mean semi-irregular vector for $T$, it is clear that $(x, 0)$ is a mean Li-Yorke pair for $T$.

(ii) $\Rightarrow$ (i): If $(x, y)$ is a mean Li-Yorke pair for $T$, then $u:=x-y$ is an absolutely mean semi-irregular vector for $T$. Hence, it follows easily that $\{\lambda u: \lambda \in \mathbb{K}\}$ is an uncountable mean Li-Yorke set for $T$.

(i) $\Rightarrow(\mathrm{v})$ : Let $(x, y)$ be a mean Li-Yorke pair for $T$ and put $u:=x-y$. Let

$$
Y:=\overline{\operatorname{span}}(\operatorname{Orb}(u, T)),
$$


which is an infinite-dimensional closed $T$-invariant subspace of $X$. Consider the operator $S \in L(Y)$ obtained by restricting $T$ to $Y$. We claim that $S$ is not absolutely Cesàro bounded. Indeed, suppose that this is not the case and let $C>0$ be such that

$$
\sup _{N \in \mathbb{N}} \frac{1}{N} \sum_{j=1}^{N}\left\|S^{j} z\right\| \leq C\|z\| \quad \text { for all } z \in Y .
$$

Since $(x, y)$ is a mean Li-Yorke pair for $T$, we have that

$$
\liminf _{N \rightarrow \infty} \frac{1}{N} \sum_{j=1}^{N}\left\|S^{j} u\right\|=0 \quad \text { and } \quad \limsup _{N \rightarrow \infty} \frac{1}{N} \sum_{j=1}^{N}\left\|S^{j} u\right\|>\varepsilon,
$$

for some $\varepsilon>0$. Let $\delta>0$ be so small that

$$
\limsup _{N \rightarrow \infty} \frac{1}{N} \sum_{j=1}^{N}\left\|S^{j} u\right\|>\delta+\varepsilon
$$

There are $N \in \mathbb{N}$ and $M>N$ such that

$$
\frac{1}{N} \sum_{j=1}^{N}\left\|S^{j} u\right\|<\delta \quad \text { and } \quad \frac{1}{M} \sum_{j=1}^{M}\left\|S^{j} u\right\|>\delta+\varepsilon .
$$

Let $K \in\{1, \ldots, N\}$ be the largest integer such that $\left\|S^{K} u\right\|<\delta$. Then

$$
\frac{1}{K} \sum_{j=1}^{K}\left\|S^{j} u\right\|<\delta
$$

Since

$$
\begin{aligned}
\delta+\varepsilon & <\frac{1}{M} \sum_{j=1}^{M}\left\|S^{j} u\right\| \\
& =\frac{K}{M} \frac{1}{K} \sum_{j=1}^{K}\left\|S^{j} u\right\|+\frac{M-K}{M} \frac{1}{M-K} \sum_{j=K+1}^{M}\left\|S^{j} u\right\| \\
& <\delta+\frac{1}{M-K} \sum_{j=K+1}^{M}\left\|S^{j} u\right\|
\end{aligned}
$$

we have that

$$
\varepsilon<\frac{1}{M-K} \sum_{j=K+1}^{M}\left\|S^{j} u\right\|=\frac{1}{M-K} \sum_{j=1}^{M-K}\left\|S^{j} S^{K} u\right\| \leq C\left\|S^{K} u\right\|<C \delta .
$$

Hence, $C>\varepsilon / \delta$. Since $\delta>0$ can be chosen arbitrarily close to 0 , we have a contradiction. This proves that $S$ is not absolutely Cesàro bounded. Therefore, the set

$$
A:=\left\{z \in Y: \sup _{N \in \mathbb{N}} \frac{1}{N} \sum_{j=1}^{N}\left\|S^{j} z\right\|=\infty\right\}
$$


is residual in $Y$ by Theorem 4 . On the other hand, let

$$
B:=\left\{z \in Y: \inf _{N \in \mathbb{N}} \frac{1}{N} \sum_{j=1}^{N}\left\|S^{j} z\right\|=0\right\} .
$$

Since

$$
B=\bigcap_{k=1}^{\infty} \bigcup_{N=1}^{\infty}\left\{z \in Y: \frac{1}{N} \sum_{j=1}^{N}\left\|S^{j} z\right\|<\frac{1}{k}\right\},
$$

we have that $B$ is a $G_{\delta}$ set. Since there is an increasing sequence $\left(N_{k}\right)$ in $\mathbb{N}$ such that

$$
\lim _{k \rightarrow \infty} \frac{1}{N_{k}} \sum_{j=1}^{N_{k}}\left\|S^{j} u\right\|=0
$$

it follows that $\operatorname{span}(\operatorname{Orb}(u, S))$ is contained in $B$. Hence, $B$ is a residual set in $Y$. Our conclusion is that $A \cap B$ is a residual set in $Y$, which is formed by absolutely mean irregular vectors for $S$ (hence for $T$ ).

Corollary 6. No absolutely Cesàro bounded operator on a Banach space is mean Li-Yorke chaotic.

The proof of Theorem 5 clearly implies the following result:

Theorem 7. For every $T \in L(X)$, the set of all absolutely mean irregular vectors for $T$ is dense in the set of all absolutely mean semi-irregular vectors for $T$.

Definition 8. We say that $T \in L(X)$ satisfies the Mean Li-Yorke Chaos Criterion (MLYCC) if there exists a subset $X_{0}$ of $X$ with the following properties:

(a) $\liminf _{N \rightarrow \infty} \frac{1}{N} \sum_{j=1}^{N}\left\|T^{j} x\right\|=0$ for every $x \in X_{0}$;

(b) there are sequences $\left(y_{k}\right)$ in $\overline{\operatorname{span}\left(X_{0}\right)}$ and $\left(N_{k}\right)$ in $\mathbb{N}$ such that

$$
\frac{1}{N_{k}} \sum_{j=1}^{N_{k}}\left\|T^{j} y_{k}\right\|>k\left\|y_{k}\right\| \text { for every } k \in \mathbb{N} .
$$

Let us now prove that this criterion characterizes mean Li-Yorke chaos.

Theorem 9. An operator $T \in L(X)$ is mean Li-Yorke chaotic if and only if it satisfies the $M L Y C C$.

Proof. $(\Rightarrow)$ : By Theorem 5, $T$ has an absolutely mean irregular vector $x$. So, it is enough to consider $X_{0}:=\{x\}$.

$(\Leftarrow)$ : If $T$ has an absolutely mean semi-irregular vector, then $T$ is mean Li-Yorke chaotic by Theorem 5 . So, let us assume that this is not the case. Then, (a) implies that

$$
\lim _{N \rightarrow \infty} \frac{1}{N} \sum_{j=1}^{N}\left\|T^{j} x\right\|=0 \text { for every } x \in X_{0} .
$$


Let

$$
Y:=\overline{\left\{x \in X: \lim _{N \rightarrow \infty} \frac{1}{N} \sum_{j=1}^{N}\left\|T^{j} x\right\|=0\right\}}
$$

which is a closed $T$-invariant subspace of $X$. Consider the operator $S \in L(Y)$ obtained by restricting $T$ to $Y$. Since the sequence $\left(y_{k}\right)$ lies in $Y$, (b) implies that $S$ is not absolutely Cesàro bounded. Hence, by Theorem 4, the set

$$
A:=\left\{y \in Y: \sup _{N \in \mathbb{N}} \frac{1}{N} \sum_{j=1}^{N}\left\|S^{j} y\right\|=\infty\right\}
$$

is residual in $Y$. On the other hand, since the set

$$
B:=\left\{y \in Y: \inf _{N \in \mathbb{N}} \frac{1}{N} \sum_{j=1}^{N}\left\|S^{j} y\right\|=0\right\}
$$

is obviously dense in $Y$, it is residual in $Y$. Thus, $A \cap B$ is residual in $Y$, which proves the existence of an absolutely mean irregular vector for $T$. By Theorem 5, we conclude that $T$ is mean Li-Yorke chaotic.

Example 10. There are mixing operators that are not mean Li-Yorke chaotic.

Indeed, in [13, Example 23] it was given some examples of mixing operators that are absolutely Cesàro bounded. One of them was taken from [33], and appears in the PhD Thesis of Beltrán Meneu with a proof provided by the third author (Theorem 3.7.3 in [7]). By Corollary 6, these operators are not mean Li-Yorke chaotic.

Example 11. There are Devaney chaotic operators that are not mean Li-Yorke chaotic.

Indeed, let $T$ be the Devaney chaotic operator constructed by Menet [34]. Suppose that $x \neq 0$ and

$$
\liminf _{N \rightarrow \infty} \frac{1}{N} \sum_{j=1}^{N}\left\|T^{j} x\right\|=0
$$

For every $\delta>0$, since

$$
\frac{\operatorname{card}\left(\left\{1 \leq j \leq N:\left\|T^{j} x\right\| \geq \delta\right\}\right)}{N} \leq \frac{1}{N} \sum_{j=1}^{N} \frac{\left\|T^{j} x\right\|}{\delta}=\frac{1}{\delta} \frac{1}{N} \sum_{j=1}^{N}\left\|T^{j} x\right\|,
$$

we have that

$$
\underline{\operatorname{dens}}\left(\left\{n \geq 0:\left\|T^{n} x\right\| \geq \delta\right\}\right)=0 .
$$

This contradicts Claim 10 in [34]. Hence, $T$ is not mean Li-Yorke chaotic.

Example 12. There are frequently hypercyclic operators that are not mean Li-Yorke chaotic.

Indeed, Bayart and Ruzsa [6, Section 6] proved that there exists a frequently hypercyclic operator (thus this operator is $\mathrm{DC} 2 \frac{1}{2}$ by [13, Theorem 13]) such that the orbit of no $x \neq 0$ is distributionally near of 0 . Hence, it is not DC1 and not mean Li-Yorke chaotic [13, Proposition 20(a)]. 
Example 13. There are distributionally chaotic operators that are not mean Li-Yorke chaotic.

We recall the following result that was obtained in [13]: If $X=c_{0}(\mathbb{N})\left(X=c_{0}(\mathbb{Z})\right)$ or $X=\ell^{p}(\mathbb{N})\left(X=\ell^{p}(\mathbb{Z})\right)$ for some $1 \leq p \leq \infty$, then there exists an (invertible) operator $T \in L(X)$ which is distributionally chaotic and satisfies

$$
\lim _{N \rightarrow \infty} \frac{1}{N} \sum_{j=1}^{N}\left\|T^{j} x\right\|=\infty \quad \text { for all } x \in X \backslash\{0\} .
$$

Since these operators do not have an absolutely mean irregular vector, Theorem 5 guarantees that they are not mean Li-Yorke chaotic. Since DC1 and DC2 are equivalent for operators on Banach spaces [13, Theorem 2], this implies that the notions of mean Li-Yorke chaos and DC2 are not equivalent for operators on Banach spaces, contrary to what happens in the context of topological dynamics on compact metric spaces [20].

Remark 14. The above examples are Li-Yorke chaotic, but not mean Li-Yorke chaotic.

We recall that an operator $T$ on a separable Banach space $X$ is said to satisfy the Frequent Hypercyclicity Criterion (FHC) if there exist a dense subset $X_{0}$ of $X$ and a map $S: X_{0} \rightarrow X_{0}$ such that, for any $x \in X_{0}$,

- $\sum_{n=0}^{\infty} T^{n} x$ converges unconditionally,

- $\sum_{n=0}^{\infty} S^{n} x$ converges unconditionally,

- $T S x=x$.

If $T$ satisfies this criterion, then $T$ is frequently hypercyclic, Devaney chaotic, mixing and distributionally chaotic $[11,16]$. Since we have just seen that there are examples of frequently hypercyclic operators, Devaney chaotic operators, mixing operators and distributionally chaotic operators that are not mean Li-Yorke chaotic, it is natural to ask if the Frequent Hypercyclicity Criterion implies mean Li-Yorke chaos. Let us now see that the answer is yes.

Proposition 15. If $T \in L(X)$ satisfies the Frequent Hypercyclicity Criterion, then $T$ has a residual set of absolutely mean irregular vectors.

Proof. If $T$ satisfies the Frequent Hypercyclicity Criterion, then the set

$$
\left\{x \in X: \lim _{n \rightarrow \infty}\left\|T^{n} x\right\|=0\right\}
$$

is dense in $X$ and $T$ is distributionally chaotic. Hence, we can apply [13, Theorem 27].

Concerning Figure 1, it is easy to construct a mean Li-Yorke chaotic operator which is not hypercyclic. Indeed, let $T \in L(X)$ be any mean Li-Yorke chaotic operator and consider the operator $T \oplus I$ on $X \oplus X$, where $I$ denotes the identity operator on $X$. However, the following question remains open.

Question 16. Is there a Banach (or Hilbert) space operator which is mean Li-Yorke chaotic but is not distributionally chaotic? 


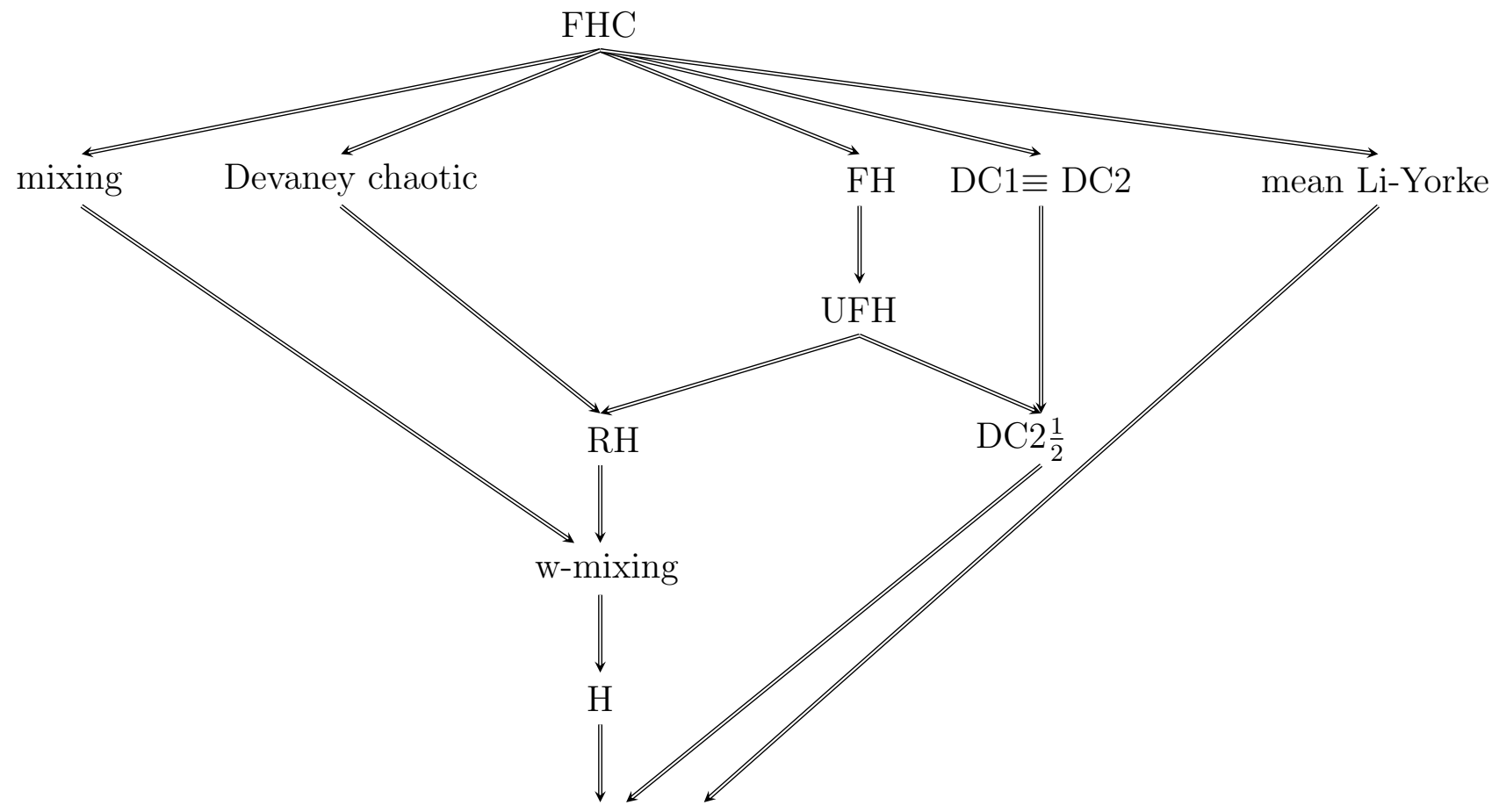

Li-Yorke chaotic

Figure 1: Implications between different definitions related with hypercyclicity and chaos for operators on Banach spaces.

Related to Question 16, we have the following result from [13]: If $X=c_{0}(\mathbb{N})$ (X= $c_{0}(\mathbb{Z})$ ) or $X=\ell^{p}(\mathbb{N})\left(X=\ell^{p}(\mathbb{Z})\right)$ for some $1 \leq p \leq \infty$, then there exists an (invertible) operator $T \in L(X)$ which admits an absolutely mean irregular vector whose orbit is not distributionally unbounded.

In particular, this shows that an absolutely mean irregular vector is not necessarily distributionally irregular. However, we don't know if the operator admits other vectors with distributionally unbounded orbit, or if it has a distributionally irregular vector.

\section{Densely mean Li-Yorke chaotic operators}

Let us now show that, in separable spaces, dense mean Li-Yorke chaos is equivalent to the existence of a residual set of absolutely mean irregular vectors.

Theorem 17. Assume $X$ separable. For every $T \in L(X)$, the following assertions are equivalent:

(i) $T$ is densely mean Li-Yorke chaotic;

(ii) $T$ has a dense set of mean Li-Yorke pairs;

(iii) $T$ has a residual set of mean Li-Yorke pairs;

(iv) Thas a dense set of absolutely mean semi-irregular vectors;

(v) $T$ has a dense set of absolutely mean irregular vectors;

(vi) T has a residual set of absolutely mean irregular vectors. 
Proof. (ii) $\Leftrightarrow$ (iv): It follows easily from the fact that $(x, y)$ is a mean Li-Yorke pair for $T$ if and only if $x-y$ is an absolutely mean semi-irregular vector for $T$.

(iv) $\Leftrightarrow(\mathrm{v})$ : It follows from Theorem 7 .

(v) $\Leftrightarrow$ (vi): The set $R$ of all absolutely mean irregular vectors for $T$ is the intersection of the sets

$$
A:=\left\{x \in X: \sup _{N \in \mathbb{N}} \frac{1}{N} \sum_{j=1}^{N}\left\|T^{j} x\right\|=\infty\right\} \text { and } B:=\left\{x \in X: \inf _{N \in \mathbb{N}} \frac{1}{N} \sum_{j=1}^{N}\left\|T^{j} x\right\|=0\right\} .
$$

It follows from Theorem 4 that $A$ is residual in $X$ whenever it is nonempty. And we saw in the proof of Theorem 5 that $B$ is residual in $X$ whenever it is dense in $X$. Thus, $R$ is residual in $X$ whenever it is dense in $X$.

(vi) $\Rightarrow$ (iii): If $\left(U_{j}\right)$ is a sequence of dense open sets in $X$ such that every vector in $\bigcap U_{j}$ is absolutely mean irregular for $T$, then the sets $V_{j}:=\left\{(x, y) \in X \times X: x-y \in U_{j}\right\}$ are open and dense in $X \times X$, and every point in $\bigcap V_{j}$ is a mean Li-Yorke pair for $T$.

The implications (iii) $\Rightarrow$ (ii) and (i) $\Rightarrow$ (ii) are obvious.

(vi) $\Rightarrow$ (i): Let $R$ be the set of all absolutely mean irregular vectors for $T$ and let $\left(y_{j}\right)$ be a dense sequence in $X$. Let $D:=\mathbb{Q}$ or $\mathbb{Q}+i \mathbb{Q}$, depending on whether the scalar field $\mathbb{K}$ is $\mathbb{R}$ or $\mathbb{C}$, respectively. Since $R$ is residual in $X$, we can choose inductively linearly independent vectors $x_{1}, x_{2}, x_{3}, \ldots \in X$ such that $x_{1} \in B\left(y_{1} ; 1\right) \cap R$ and

$$
x_{n+1} \in B\left(y_{n+1} ; \frac{1}{n+1}\right) \cap \bigcap_{\left(\alpha_{1}, \ldots, \alpha_{n}\right) \in D^{n}}\left(\alpha_{1} x_{1}+\cdots+\alpha_{n} x_{n}+R\right) \quad(n \in \mathbb{N}) .
$$

Hence,

$$
M:=\left\{\alpha_{1} x_{1}+\cdots+\alpha_{m} x_{m}: m \geq 1 \text { and } \alpha_{1}, \ldots, \alpha_{m} \in D\right\}
$$

is a dense $D$-vector subspace of $X$ consisting (up to 0 ) of absolutely mean irregular vectors for $T$. In particular, $M$ is a dense mean Li-Yorke set for $T$. Since $M$ is countable, we need to enlarge $M$ in order to obtain an uncountable dense mean Li-Yorke set for $T$. Let

$$
N:=\left\{\alpha_{2} x_{2}+\cdots+\alpha_{m} x_{m}: m \geq 2 \text { and } \alpha_{2}, \ldots, \alpha_{m} \in D\right\} .
$$

For each $y \in N \backslash\{0\}$, let $A_{y}:=\left\{\lambda \in \mathbb{K}: y-\lambda x_{1}\right.$ is absolutely mean irregular for $\left.T\right\}$. Since $A_{y}$ is a $G_{\delta}$ set in $\mathbb{K}$ containing $D, A_{y}$ is residual in $\mathbb{K}$. Thus, $A:=\bigcap_{y \in N \backslash\{0\}} A_{y}$ is also a residual set in $\mathbb{K}$ containing $D$. By Zorn's Lemma, there is a maximal $D$-vector subspace $H$ of $\mathbb{K}$ such that $D \subset H \subset A$. If $H$ were countable, then

$$
B:=\bigcap_{\beta \in D \backslash\{0\}} \bigcap_{\alpha \in H} \beta(\alpha+A)
$$

would be residual in $\mathbb{K}$. By choosing $\gamma \in B \backslash H$, we would have that $H^{\prime}:=H+\{\beta \gamma$ : $\beta \in D\}$ is a $D$-vector subspace of $\mathbb{K}$ satisfying $D \subset H^{\prime} \subset A$ and $H \subsetneq H^{\prime}$, which would contradict the maximality of $H$. Thus, $M^{\prime}:=\left\{\alpha x_{1}: \alpha \in H\right\}+N$ is the uncountable mean Li-Yorke set for $T$ we were looking for.

Remark 18. Note that in the previous theorem the separability of $X$ was used only in the proof that $(\mathrm{vi}) \Rightarrow(\mathrm{i})$. The equivalences

$$
\text { (ii) } \Leftrightarrow \text { (iii) } \Leftrightarrow \text { (iv) } \Leftrightarrow \text { (v) } \Leftrightarrow \text { (vi) }
$$

are valid for any Banach space. 
In view of Theorem 5 , it is true that an operator $T \in L(X)$ is mean Li-Yorke chaotic if and only if there is a mean Li-Yorke set for $T$ which is a one-dimensional subspace of $X$. Nevertheless, we shall now show that the chaotic behaviour always occurs in a much larger subspace of $X$.

Theorem 19. Every mean Li-Yorke chaotic operator $T \in L(X)$ is densely mean Li-Yorke chaotic on some infinite-dimensional closed $T$-invariant subspace of $X$.

Proof. By Theorem 5, the restriction of $T$ to a certain infinite-dimensional closed $T$ invariant subspace $Y$ of $X$ has a residual set of absolutely mean irregular vectors. The proof of Theorem 5 actually constructs a separable such $Y$. Hence, we can apply Theorem 17 and conclude that $T$ is densely mean Li-Yorke chaotic on $Y$.

Definition 20. We say that $T \in L(X)$ satisfies the Dense Mean Li-Yorke Chaos Criterion (DMLYCC) if there exists a dense subset $X_{0}$ of $X$ with properties (a) and (b) of Definition 8.

Theorem 21. Assume $X$ separable. An operator $T \in L(X)$ is densely mean Li-Yorke chaotic if and only if it satisfies the DMLYCC.

Proof. $(\Rightarrow)$ : By Theorem 17, $T$ has a dense set $X_{0}$ of absolutely mean irregular vectors. Clearly, $X_{0}$ satisfies properties (a) and (b) of Definition 8.

$(\Leftarrow)$ : Since $X_{0}$ is dense in $X$, condition (a) implies that the set

$$
B:=\left\{x \in X: \inf _{N \in \mathbb{N}} \frac{1}{N} \sum_{j=1}^{N}\left\|T^{j} x\right\|=0\right\}
$$

is residual in $X$. By (b), $T$ is not absolutely Cesàro bounded. Hence, by Theorem 4, the set

$$
A:=\left\{x \in X: \sup _{N \in \mathbb{N}} \frac{1}{N} \sum_{j=1}^{N}\left\|T^{j} x\right\|=\infty\right\}
$$

is also residual in $X$. Thus, $T$ has a residual set of absolutely mean irregular vectors. By Theorem 17, we conclude that $T$ is densely mean Li-Yorke chaotic.

Theorem 22. If $T \in L(X)$ and

$$
X_{0}:=\left\{x \in X: \liminf _{N \rightarrow \infty} \frac{1}{N} \sum_{j=1}^{N}\left\|T^{j} x\right\|=0\right\}
$$

is dense in $X$, then the following assertions are equivalent:

(i) $T$ is mean Li-Yorke chaotic;

(ii) $T$ has a residual set of absolutely mean irregular vectors;

(iii) $T$ is not absolutely Cesàro bounded;

(iv) $\limsup _{N \rightarrow \infty} \frac{1}{N} \sum_{j=1}^{\infty}\left\|T^{j} y_{0}\right\|>0$ for some $y_{0} \in X$. 
Proof. (i) $\Rightarrow$ (iii): It follows from Corollary 6 .

(iii) $\Rightarrow$ (ii): Since $X_{0}$ is dense in $X$ by hypothesis, it is residual in $X$. Hence, (ii) follows from Theorem 4.

The implication (ii) $\Rightarrow$ (i) follows from Theorem 5 .

The implication (i) $\Rightarrow$ (iv) is trivial.

(iv) $\Rightarrow(\mathrm{i})$ : Suppose that $T$ is not mean Li-Yorke chaotic. By Theorem 5, there is no absolutely mean semi-irregular vector for $T$, and so

$$
X_{0}=\left\{x \in X: \lim _{N \rightarrow \infty} \frac{1}{N} \sum_{j=1}^{N}\left\|T^{j} x\right\|=0\right\} .
$$

Thus, $X_{0}$ is a residual subspace of $X$, which implies that $X_{0}=X$ and contradicts (iv).

Remark 23. In the case that the space $X$ is separable, Theorem 17 shows that condition (ii) in the above theorem can be replaced by

(ii') $T$ is densely mean Li-Yorke chaotic.

As an immediate consequence of the previous theorem, we have the following dichotomy for unilateral weighted backward shifts on Banach sequence spaces.

Corollary 24. Let $X$ be a Banach sequence space in which $\left(e_{n}\right)_{n \in \mathbb{N}}$ is a basis [26, Section 4.1]. Suppose that the unilateral weighted backward shift

$$
B_{w}\left(x_{1}, x_{2}, x_{3}, \ldots\right):=\left(w_{2} x_{2}, w_{3} x_{3}, w_{4} x_{4}, \ldots\right)
$$

is an operator on $X$. Then either

(a) $B_{w}$ is mean Li-Yorke chaotic, or

(b) $B_{w}$ is absolutely Cesàro bounded.

Another consequence of Theorem 22 is given in the following corollary. Items (a) and (b) improve Theorems 27 and 28 of [13], respectively.

Corollary 25. Let $T \in L(X)$ be such that

$$
\left\{x \in X: \liminf _{N \rightarrow \infty} \frac{1}{N} \sum_{j=1}^{N}\left\|T^{j} x\right\|=0\right\}
$$

is dense in $X$. If any of the following conditions is true:

(a) $T$ is distributionally chaotic,

(b) $T$ is Cesàro hypercyclic,

(c) $X$ is a Banach space and $\limsup _{n \rightarrow \infty} \frac{\left\|T^{n}\right\|}{n}>0$,

(d) $X$ is a Hilbert space and $\limsup _{n \rightarrow \infty} \frac{\left\|T^{n}\right\|}{n^{\frac{1}{2}}}>0$,

(e) $T$ has an eigenvalue $\lambda$ with $|\lambda| \geq 1$, 
Remark: If $\left\{x: T^{n} x \rightarrow 0\right\}$ is a dense set in $X$, then:

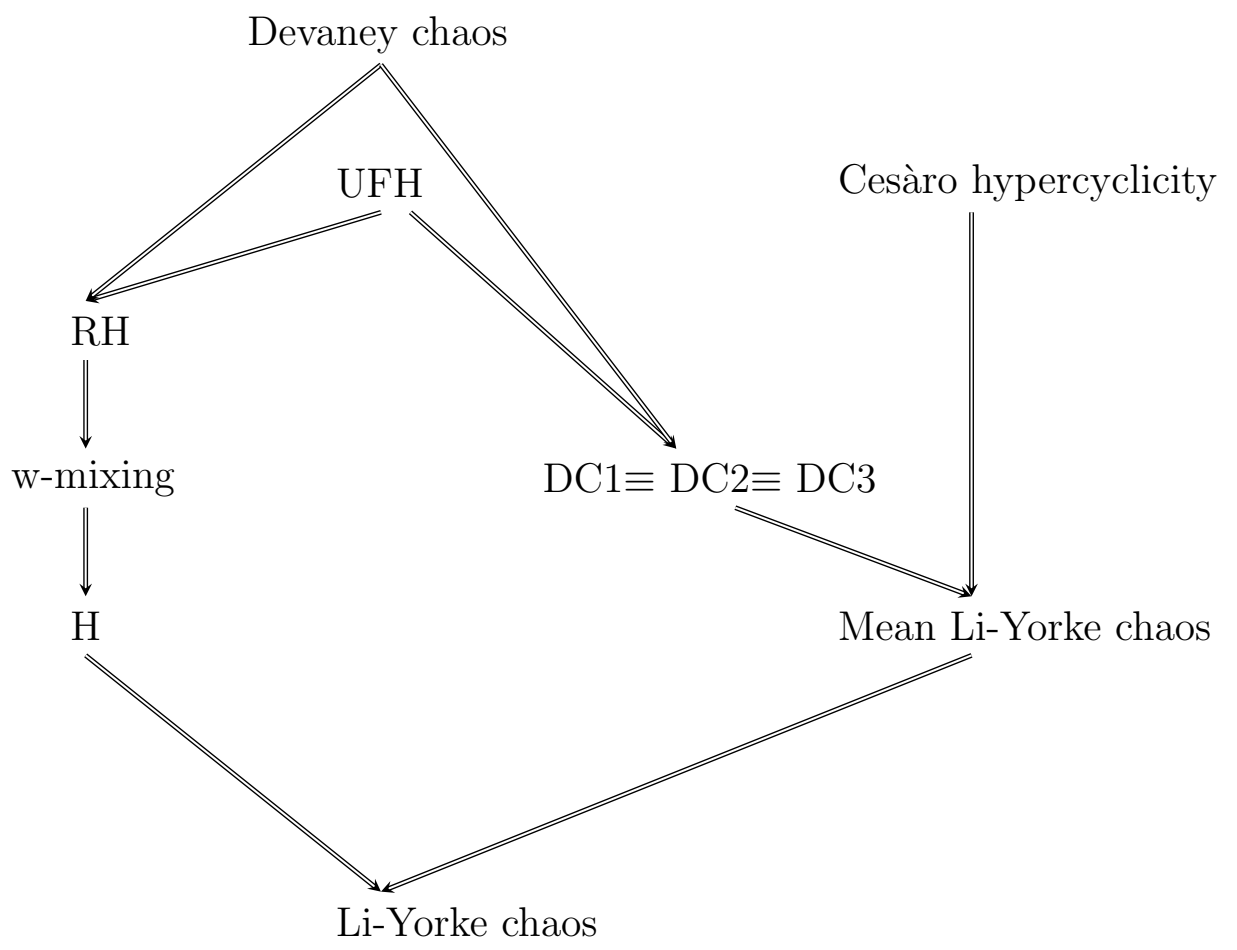

Figure 2: Implications between different definitions related with hypercyclicity and chaos for operators on Banach spaces when $\left\{x: T^{n} x \rightarrow 0\right\}$ is a dense set in $X$.

then there is a residual set of absolutely mean irregular vectors for $T$. If, in addition, $X$ is separable, then $T$ is densely mean Li-Yorke chaotic.

Proof. Any of these conditions implies that

$$
\limsup _{N \rightarrow \infty} \frac{1}{N} \sum_{j=1}^{N}\left\|T^{j} y_{0}\right\|>0 \text { for some } y_{0} \in X
$$

(for items (c) and (d) this follows from Theorem 2.4 and Corollary 2.6 of [9]). Thus, the result follows from Theorem 22 .

Example 26. There are densely mean Li-Yorke chaotic operators that are not Cesàro hypercyclic.

Indeed, let $T$ be the weighted backward shift on $\ell^{1}(\mathbb{N})$ defined by

$$
T e_{1}=0 \quad \text { and } \quad T e_{k}=\left(\frac{k}{k-1}\right) e_{k-1} \text { for } k>1 .
$$

Since $\left\|T^{n}\right\|=n+1$ for all $n \in \mathbb{N}$, Corollary 25(c) implies that $T$ is densely mean Li-Yorke chaotic. Moreover, the equalities $\left\|T^{n}\right\|=n+1(n \in \mathbb{N})$ also imply that $\left(\frac{T^{n} x}{n}\right)$ is not dense in $\ell^{1}(\mathbb{N})$, for every $x \in \ell^{1}(\mathbb{N})$. Thus, $T$ is not Cesàro hypercyclic [30].

One may think that the above example might be a good candidate for a mean Li-Yorke chaotic operator which is not distributionally chaotic, but actually this is not the case 
because of the following result which was generously provided to us by Frédéric Bayart in private communication:

In general, for $\alpha>0$ and $1 \leq p<\infty$, let $T$ be the unilateral weighted backward shift on $\ell^{p}(\mathbb{N})$ defined by

$$
T e_{1}=0 \quad \text { and } \quad T e_{k}=\left(\frac{k}{k-1}\right)^{\alpha} e_{k-1} \text { for } k>1
$$

Theorem 27. If $\alpha=\frac{1}{p}$, then $T$ has a residual set of distributionally irregular vectors.

Proof. In view of [11, Theorem 15], it is sufficient to prove that $T$ has a distributionally unbounded orbit. Let $\left\{n_{k}\right\}_{k \in \mathbb{N}}$ be an increasing sequence of natural numbers such that

$$
\frac{\left[\log \left(n_{k}\right)\right]^{1 / p}}{k^{2}} \rightarrow \infty \text { as } k \rightarrow \infty
$$

Define, for $k \geq 1$,

$$
x_{k}:=\frac{1}{k^{2}} \sum_{n=n_{k}}^{k n_{k}} \frac{1}{n^{1 / p}} e_{n} .
$$

Since

$$
\left\|x_{k}\right\|_{p}=\frac{1}{k^{2}}\left(\sum_{n=n_{k}}^{k n_{k}} \frac{1}{n}\right)^{1 / p} \leq \frac{C}{k^{2}}\left[\log \left(k n_{k}\right)-\log \left(n_{k}\right)\right]^{1 / p}=C \frac{(\log (k))^{1 / p}}{k^{2}},
$$

we may consider $x:=\sum_{k \geq 1} x_{k}$, which belongs to $\ell^{p}(\mathbb{N})$. Moreover, if we set $B_{k}:=$ $\left[n_{k},(k-1) n_{k}\right]$ and $B:=\cup_{k \in \mathbb{N}} B_{k}$, we have that $\overline{\operatorname{dens}}(B)=1$. Now, for $n \in B_{k}$,

$$
\begin{aligned}
\left\|T^{n} x\right\|_{p} & \geq\left\|T^{n} x_{k}\right\|_{p} \geq \frac{1}{k^{2}}\left\|\sum_{j=1}^{n_{k}} \frac{1}{(n+j)^{1 / p}} T^{n} e_{n+j}\right\|_{p} \\
& \geq \frac{1}{k^{2}}\left\|\sum_{j=1}^{n_{k}} \frac{1}{j^{1 / p}} e_{j}\right\|_{p} \geq \frac{1}{k^{2}}\left(\sum_{j=1}^{n_{k}} \frac{1}{j}\right)^{1 / p} \geq C \frac{\left(\log \left(n_{k}\right)\right)^{1 / p}}{k^{2}} \rightarrow \infty .
\end{aligned}
$$

Hence, $x$ has a distributionally unbounded orbit.

\section{Dense lineability of absolutely mean irregular vec- tors}

Definition 28. An absolutely mean irregular manifold for $T \in L(X)$ is a vector subspace $Y$ of $X$ such that every nonzero vector in $Y$ is absolutely mean irregular for $T$.

Such a manifold is clearly a mean Li-Yorke set for $T$. The following dichotomy gives us a sufficient condition for the existence of a dense absolutely mean irregular manifold.

Theorem 29. Assume $X$ separable. If $T \in L(X)$ and

$$
X_{0}:=\left\{x \in X: \lim _{N \rightarrow \infty} \frac{1}{N} \sum_{j=1}^{N}\left\|T^{j} x\right\|=0\right\}
$$

is dense in $X$, then either 
(a) $\lim _{N \rightarrow \infty} \frac{1}{N} \sum_{j=1}^{N}\left\|T^{j} x\right\|=0$ for every $x \in X$, or

(b) T admits a dense absolutely mean irregular manifold.

Proof. Suppose that (a) is false and let us prove (b). By Theorem 22, $T$ has an absolutely mean irregular vector. Let $C:=\|T\|>1$. Then, we can construct a sequence $\left(x_{m}\right)$ of normalized vectors in $X_{0}$ and an increasing sequence $\left(N_{m}\right)$ of positive integers so that

$$
\frac{1}{N_{m}} \sum_{i=1}^{N_{m}}\left\|T^{i} x_{m}\right\|>m(2 C)^{m} \text { and } \frac{1}{N_{m}} \sum_{i=1}^{N_{m}}\left\|T^{i} x_{k}\right\|<\frac{1}{m} \text { for } k=1, \ldots, m-1 .
$$

Given $\alpha, \beta \in\{0,1\}^{\mathbb{N}}$, we say that $\beta \leq \alpha$ if $\beta_{i} \leq \alpha_{i}$ for all $i \in \mathbb{N}$. Let $\left(r_{j}\right)$ be a sequence of positive integers such that $r_{j+1} \geq 1+r_{j}+N_{r_{j}+1}$ for all $j \in \mathbb{N}$. Let $\alpha \in\{0,1\}^{\mathbb{N}}$ be defined by $\alpha_{n}=1$ if and only if $n=r_{j}$ for some $j \in \mathbb{N}$. For each $\beta \in\{0,1\}^{\mathbb{N}}$ such that $\beta \leq \alpha$ and $\beta$ contains an infinite number of 1's, we define

$$
x_{\beta}:=\sum_{i} \frac{\beta_{i}}{(2 C)^{i}} x_{i}=\sum_{j} \frac{\beta_{r_{j}}}{(2 C)^{r_{j}}} x_{r_{j}} .
$$

Take $k \in \mathbb{N}$ with $\beta_{r_{k}}=1$. Since $\frac{1}{N_{r_{k}}} \sum_{i=1}^{N_{r_{k}}}\left\|T^{i} x_{r_{k}}\right\|>r_{k}(2 C)^{r_{k}}$ and $\frac{1}{N_{r_{k}}} \sum_{i=1}^{N_{r_{k}}}\left\|T^{i} x_{s}\right\|<\frac{1}{r_{k}}$ for each $s<r_{k}$, we have that

$$
\begin{aligned}
\frac{1}{N_{r_{k}}} \sum_{i=1}^{N_{r_{k}}}\left\|T^{i} x_{\beta}\right\| & \geq \frac{1}{(2 C)^{r_{k}}} \frac{1}{N_{r_{k}}} \sum_{i=1}^{N_{r_{k}}}\left\|T^{i} x_{r_{k}}\right\|-\frac{1}{N_{r_{k}}} \sum_{i=1}^{N_{r_{k}}} \sum_{j \neq k} \frac{\beta_{r_{j}}}{(2 C)^{r_{j}}}\left\|T^{i} x_{r_{j}}\right\| \\
& >r_{k}-\frac{1}{r_{k}} \sum_{j<k} \frac{1}{(2 C)^{r_{j}}}-\sum_{j>k} \frac{\left\|x_{r_{j}}\right\|}{2^{r_{j}}} \\
& \geq r_{k}-1 .
\end{aligned}
$$

On the other hand, since $\frac{1}{N_{r_{k}+1}} \sum_{i=1}^{N_{r_{k}+1}}\left\|T^{i} x_{s}\right\|<\frac{1}{r_{k}+1}$ for each $s<r_{k}+1$, then

$$
\begin{aligned}
\frac{1}{N_{r_{k}+1}} \sum_{i=1}^{N_{r_{k}+1}}\left\|T^{i} x_{\beta}\right\| & \leq \frac{1}{N_{r_{k}+1}} \sum_{i=1}^{N_{r_{k}+1}} \sum_{j \leq k} \frac{\beta_{r_{j}}\left\|T^{i} x_{r_{j}}\right\|}{(2 C)^{r_{j}}}+\frac{1}{N_{r_{k}+1}} \sum_{i=1}^{N_{r_{k}+1}} \sum_{j>k} \frac{\beta_{r_{j}}\left\|T^{i} x_{r_{j}}\right\|}{(2 C)^{r_{j}}} \\
& \leq \frac{1}{r_{k}+1} \sum_{j \leq k} \frac{1}{(2 C)^{r_{j}}}+\frac{1}{N_{r_{k}+1}} \sum_{i=1}^{N_{r_{k}+1}} \sum_{j>k} \frac{\left\|x_{r_{j}}\right\|}{2^{r_{j}}} \\
& <\frac{1}{r_{k}+1} .
\end{aligned}
$$

Thus, $x_{\beta}$ is an absolutely mean irregular for $T$.

Now, let $\left(w_{n}\right)$ be a dense sequence in $X_{0}$ and choose $\gamma_{n} \in\{0,1\}^{\mathbb{N}}(n \in \mathbb{N})$ such that each $\gamma_{n}$ contains an infinite number of 1's, $\gamma_{n} \leq \alpha$ for every $n \in \mathbb{N}$, and the sequences $\gamma_{n}$ have mutually disjoint supports. Define $v_{n}:=\sum_{i} \frac{\gamma_{n, i}}{(2 C)^{i}} x_{i}$ and $y_{n}:=w_{n}+\frac{1}{n} v_{n}(n \in \mathbb{N})$. 
Then $Y:=\operatorname{span}\left\{y_{n}: n \in \mathbb{N}\right\}$ is a dense subspace of $X$. Moreover, if $y \in Y \backslash\{0\}$, then we can write $y=w_{0}+\sum_{k} \frac{\rho_{k}}{(2 C)^{k}} x_{k}$, where $w_{0} \in X_{0}$ and the sequence of scalars $\left(\rho_{k}\right)$ takes only a finite number of values (each of them infinitely many times). As in the above proof we can show that the vector $v:=\sum_{k} \frac{\rho_{k}}{(2 C)^{k}} x_{k}$ is absolutely mean irregular for $T$. Since $y=w_{0}+v$ and $w_{0} \in X_{0}$, we conclude that $y$ is also absolutely mean irregular for $T$.

Here is an application of the previous theorem.

Corollary 30. If $B_{w}$ is a unilateral weighted backward shift on a Banach sequence space $X$ in which $\left(e_{n}\right)_{n \in \mathbb{N}}$ is a basis, then either

(a) $\lim _{N \rightarrow \infty} \frac{1}{N} \sum_{j=1}^{N}\left\|\left(B_{w}\right)^{j} x\right\|=0$ for every $x \in X$, or

(b) $B_{w}$ admits a dense absolutely mean irregular manifold.

Example 31. There are densely mean Li-Yorke chaotic operators that are not hypercyclic.

Indeed, let $X=c_{0}(\mathbb{N})$ or $X=\ell^{p}(\mathbb{N})$ for some $1 \leq p<\infty$. Consider the unilateral weighted backward shift $B_{w}: X \rightarrow X$ whose weight sequence is given by

$$
w:=\left(\frac{1}{2}, 2, \frac{1}{2}, \frac{1}{2}, 2,2, \frac{1}{2}, \frac{1}{2}, \frac{1}{2}, 2,2,2, \ldots\right),
$$

with successive blocks of $\frac{1}{2}$ 's and 2's. Since $\sup _{n \in \mathbb{N}} \prod_{j=1}^{n}\left|w_{j}\right|=1<\infty, B_{w}$ is not hypercyclic [26, Example 4.9]. On the other hand, if we define $x \in X$ by putting $\frac{1}{2^{n}}$ in the position of the last 2 in the $n^{\text {th }}$ block of 2's, for each $n \in \mathbb{N}$, and 0 otherwise, then

$$
\left\|\left(B_{w}\right)^{n} x\right\| \geq 1 \text { for all } n \in \mathbb{N} .
$$

Hence, Corollary 30 guarantees that $B_{w}$ is densely mean Li-Yorke chaotic.

Remark 32. In Corollary 25, if $X$ is separable and we assume the stronger property that

$$
\left\{x \in X: \lim _{N \rightarrow \infty} \frac{1}{N} \sum_{j=1}^{N}\left\|T^{j} x\right\|=0\right\}
$$

is dense in $X$, then we can conclude that $T$ has a dense absolutely mean irregular manifold.

\section{Generically mean Li-Yorke chaotic operators}

We have the following characterizations of generic mean Li-Yorke chaos.

Theorem 33. For every $T \in L(X)$, the following assertions are equivalent:

(i) $T$ is generically mean Li-Yorke chaotic;

(ii) Every non-zero vector is absolutely mean semi-irregular for $T$;

(iii) $X$ is a mean Li-Yorke set for $T$.

The proof is analogous to that of [12, Theorem 34]. 
Definition 34. We say that an operator $T \in L(X)$ is completely absolutely mean irregular if every vector $x \in X \backslash\{0\}$ is absolutely mean irregular for $T$.

Thus, every completely absolutely mean irregular operator is generically mean LiYorke chaotic. The converse is not true in general (see Remark 40).

Our next goal is to construct an invertible hypercyclic operator $T$ such that both $T$ and $T^{-1}$ are completely absolutely mean irregular. The construction is a modification of the type of examples of completely distributionally irregular operators provided in [33]. We first recall one of the main results in [33].

Theorem 35. [33, Thm. 3.1] Let $v=\left(v_{j}\right)_{j \in \mathbb{Z}}$ be a weight sequence that satisfies the following conditions:

1. there are sequences of integers $\left(n_{j}\right)_{j \in \mathbb{Z}}$ and $\left(m_{j}\right)_{j \in \mathbb{Z}}$ with $n_{j}<m_{j}<n_{j+1}, j \in \mathbb{Z}$, and $M>1$ such that $M v_{m_{-k}} \geq v_{j}$ for every $j \in\left[m_{-k}, m_{k-1}\right], k \in \mathbb{N}$, and if we consider

$$
\left.\left.S_{k}:=\sup \left\{\frac{v_{j}}{v_{j-1}} ; j \notin\right] m_{-k}, m_{k-1}\right]\right\}, \quad k \in \mathbb{N},
$$

then for every $\varepsilon>0$ we find $k \in \mathbb{N}$ with $v_{n_{k}}<\varepsilon$ and

$$
S_{k}^{k\left(n_{k}-m_{-k}\right)} \leq \min \left\{M, \frac{\min \left\{v_{i} ; m_{-k} \leq i \leq m_{k-1}\right\}}{v_{n_{k}}}\right\},
$$

2. for every $N \in \mathbb{N}$, there exists $k \in \mathbb{N}$ such that $v_{j}>N$, for $k \leq j \leq N k$.

Then the forward shift $T: \ell^{p}(v, \mathbb{Z}) \rightarrow \ell^{p}(v, \mathbb{Z})$ is completely distributionally irregular.

Actually, it was shown that, given an arbitrary non-zero vector $x \in \ell^{p}(v, \mathbb{Z})$, and an arbitrary $\delta>0$, there is $k \in \mathbb{N}$ as big as we want such that

$$
\text { (I1) } \quad\left\|B^{l} x\right\|^{p}<\delta \text { for any } l \in\left[n_{k}-m_{-k}, k\left(n_{k}-m_{-k}\right)\right] .
$$

The type of examples that we will consider involve the inverse too, and we also need to recall the following result:

Corollary 36. [33, Cor. 3.3] Let $v=\left(v_{j}\right)_{j \in \mathbb{Z}}$ be a weight sequence that satisfies the following conditions:

1. there are sequences of integers $\left(n_{j}\right)_{j \in \mathbb{Z}}$ and $\left(m_{j}\right)_{j \in \mathbb{Z}}$ with $n_{j}<m_{j}<n_{j+1}, j \in \mathbb{Z}$, and $M>1$ such that $M v_{m_{k}} \geq v_{j}$ for every $j \in\left[m_{-k}, m_{k}\right], k \in \mathbb{N}$, and if we consider

$$
\left.\left.s_{k}:=\inf \left\{\frac{v_{j}}{v_{j-1}} ; j \notin\right] m_{-k}, m_{k}\right]\right\}, k \in \mathbb{N},
$$

then for every $\varepsilon>0$ we find $k \in \mathbb{N}$ with $v_{n_{-k}}<\varepsilon$ and

$$
s_{k}^{k\left(n_{-k}-m_{k}\right)} \leq \min \left\{M, \frac{\min \left\{v_{i} ; m_{-k} \leq i \leq m_{k}\right\}}{v_{n_{-k}}}\right\},
$$

2. for every $N \in \mathbb{N}$, there exits $k \in \mathbb{N}$ such that $v_{j}>N$, for $-N k \leq j \leq-k$.

Then the backward shift $B=T^{-1}: \ell^{p}(v, \mathbb{Z}) \rightarrow \ell^{p}(v, \mathbb{Z})$ is completely distributionally irregular. 
In this case, it turns out that, given an arbitrary non-zero vector $x \in \ell^{p}(v, \mathbb{Z})$, and an arbitrary $\delta>0$, there is $k \in \mathbb{N}$ as big as we want such that

(I2) $\left\|B^{l} x\right\|^{p}<\delta$ for any $l \in\left[m_{k}-n_{-k}, k\left(m_{k}-n_{-k}\right)\right]$.

These results allow us to provide examples of completely absolutely mean irregular operators, which are a modification of Example 3.5 of [33]. The sequences of integers $\left(n_{j}\right)_{j \in \mathbb{Z}}$ and $\left(m_{j}\right)_{j \in \mathbb{Z}}$ with $n_{j}<m_{j}<n_{j+1}, j \in \mathbb{Z}$, are such that for every $k \in \mathbb{Z}$ we have

$$
v_{j-1} \leq v_{j} \text { when } n_{k}<j \leq m_{k} \text {, and } v_{j-1} \geq v_{j} \text { when } m_{k}<j \leq n_{k+1} .
$$

In other words, the positions $v_{m_{k}}$ represent "hills" of the weight sequence, and the positions $v_{n_{k}}$ are "valleys".

Theorem 37. There exists a bilateral shift $T$ on $\ell^{p}(v, \mathbb{Z})$ such that both $T$ and $T^{-1}$ are completely absolutely mean irregular, completely distributionally irregular and hypercyclic.

Proof. We first consider

(a) $n_{0}=-1, m_{0}=1, n_{1}=4, m_{-1}=-4, v_{n_{0}}=1, v_{m_{0}}=2^{1 / 4}, v_{n_{1}}=2^{-1 / 3}, v_{m_{-1}}=2^{1 / 4}$, and

(b) $m_{k}=-n_{-k}, k \in \mathbb{Z}, v_{n_{k}}=(2 k)^{-1 / 3}, v_{m_{k}}=(k+2)^{1 / 4}, v_{n_{-k}}=(2 k+1)^{-1 / 3}, v_{m_{-k}}=$ $(k+1)^{1 / 4}, k \in \mathbb{N}, v_{i} / v_{i-1}=v_{j} / v_{j-1}$ if $\left.\left.i, j \in\right] n_{k}, m_{k}\right]$, or if $\left.\left.i, j \in\right] m_{k-1}, n_{k}\right], k \in \mathbb{Z}$, and

(c) $m_{k}-n_{k}>2\left(m_{k-1}-n_{k-1}\right), n_{k+1}-m_{k}>2\left(n_{k}-m_{k-1}\right), k \in \mathbb{N}$.

We will check that the hypotheses of Theorem 35 and Corollary 36 are satisfied. Condition (b) gives $\frac{\min \left\{v_{i} ; m_{-k} \leq i \leq m_{k-1}\right\}}{v_{n_{k}}}=\frac{v_{n_{-k+1}}}{v_{n_{k}}}=\left(\frac{2 k}{2 k-1}\right)^{1 / 3}$ for every $k \in \mathbb{N}$, and the supremum of the slope of $v$ outside the interval $\left[m_{-k}, m_{k-1}\right]$ is $S_{k}=v_{j} / v_{j-1}$ for any $n_{k}<j \leq m_{k}, k \in \mathbb{N}$. We set $M=2$ and, since $S_{k}^{m_{k}-n_{k}}=\frac{v_{m_{k}}}{v_{n_{k}}}=(2 k)^{1 / 3}(k+2)^{1 / 4}$, we need that

$$
S_{k}^{k\left(n_{k}-m_{-k}\right)}=\left((2 k)^{2 / 3}(k+2)^{1 / 2}\right)^{\frac{k n_{k}}{\left(m_{k}-n_{k}\right)}} \leq \frac{\min \left\{v_{i} ; m_{-k} \leq i \leq m_{k-1}\right\}}{v_{n_{k}}}=\left(\frac{2 k}{2 k-1}\right)^{1 / 3} .
$$

This can be obtained for, e.g., $m_{k}=\left(16 k^{3}+1\right) n_{k}, k \in \mathbb{N}$. Indeed, for this selection and for any $k \geq 2$ (the case $k=1$ trivially satisfies the above inequality), we have

$$
S_{k}^{k\left(n_{k}-m_{-k}\right)}<(2 k(k+2))^{1 / 24 k^{2}} \leq(2 k)^{1 / 12 k^{2}} \leq\left(\frac{2 k}{2 k-1}\right)^{1 / 3} .
$$

Thus, $T$ satisfies $(I 1)$. If $l \leq n_{k}-m_{-k}$, then

$$
\left\|T^{l} x\right\| \leq \frac{v_{m_{k}}}{v_{n_{k}}}\|x\| \leq(2 k)^{2 / 3}\|x\|,
$$

which yields that, given an arbitrary non-zero vector $x \in \ell^{p}(v, \mathbb{Z})$ and an arbitrary $\delta>0$, there is $k \in \mathbb{N}$ as big as we want such that

$$
\frac{1}{N} \sum_{j=1}^{N}\left\|T^{l} x\right\|<\frac{(2 k)^{2 / 3}}{k+1}\|x\|+\delta,
$$


for $N=k\left(n_{k}-m_{-k}\right)$, and we obtain that $T$ is completely absolutely mean irregular as soon as we show that condition (2) in Theorem 35 is satisfied. For this, we notice that

$$
v_{r}=v_{m_{k}} s_{k}^{r-m_{k}} \geq(k+2)^{1 / 4} s_{k}^{2 k m_{k}}>(k+2)^{1 / 4}\left(\frac{2 k}{2 k+1}\right)^{1 / 3} \quad \text { if } \quad m_{k} \leq r \leq(2 k+1) m_{k}
$$

which yields that $T$ is completely absolutely mean irregular.

Analogously, $\frac{\min \left\{v_{i} ; m_{-k} \leq i \leq m_{k}\right\}}{v_{n_{-k}}}=\frac{v_{n_{k}}}{v_{n_{-k}}}=\left(\frac{2 k+1}{2 k}\right)^{1 / 3}$ for every $k \in \mathbb{N}$, and the infimum of the slope of $v$ outside the interval $\left[m_{-k}, m_{k}\right]$ is $s_{k}=v_{j} / v_{j-1}$ for any $m_{k}<j \leq n_{k+1}, k \in \mathbb{N}$. Again, we set $M=2$ and, since $s_{k}^{m_{k}-n_{k+1}}=\left((2 k+2)^{1 / 3}(k+2)^{1 / 4}\right)$, we need that

$$
s_{k}^{k\left(n_{-k}-m_{k}\right)}=s_{k}^{-2 k m_{k}}=\left((2 k+2)^{2 / 3}(k+2)^{1 / 2}\right)^{\frac{k m_{k}}{\left(n_{k+1}-m_{k}\right)}} \leq\left(\frac{2 k+1}{2 k}\right)^{1 / 3},
$$

which is easily satisfied if we set, e.g., $n_{k+1}=\left(16 k^{3}+1\right) m_{k}, k \in \mathbb{N}$. Thus, $B=T^{-1}$ satisfies (I2). If $l \leq m_{k}-n_{-k}$, then

$$
\left\|B^{l} x\right\| \leq \frac{v_{m_{k}}}{v_{n_{k}}}\|x\| \leq(2 k)^{2 / 3}\|x\| .
$$

As before, we obtain that $B=T^{-1}$ is completely absolutely mean irregular in case that condition (2) in Corollary 36 is satisfied. Indeed, we easily have that

$$
v_{r}>(k+1)^{1 / 4}\left(\frac{2 k}{2 k+1}\right)^{1 / 3} \text { if }(2 k+1) m_{-k} \leq r \leq m_{-k}
$$

which implies condition (2).

For the hypercyclicity of $T$, since it is invertible, it suffices to show that there is an increasing sequence $\left(j_{k}\right)_{k}$ in $\mathbb{N}$ such that $\lim _{k} v_{j_{k}}=\lim _{k} v_{-j_{k}}=0$ (See Theorem 3.2 in $[22])$. Let $j_{k}:=\left(m_{k}+n_{k}\right) / 2=\left(8 k^{3}+1\right) n_{k}$. We have

$$
v_{j_{k}}=S_{k}^{j_{k}-n_{k}} v_{n_{k}}=\left((2 k)^{1 / 6}(k+2)^{1 / 8}\right) \frac{1}{(2 k)^{1 / 3}},
$$

that tends to 0 as $k$ goes to infinity. Note that $R_{k}=v_{j} / v_{j-1}$ has the same value for any $\left.j \in] n_{-k}, m_{-k}\right]$, and thus for all $k \in \mathbb{N}$ we have $R_{k}<S_{k}$ and

$$
v_{-j_{k}}=R_{k}^{-j_{k}-n_{-k}} v_{n_{-k}}<S_{k}^{m_{k}-j_{k}} \frac{1}{(2 k+1)^{1 / 3}} .
$$

Therefore, $\lim _{k} v_{j_{k}}=\lim _{k} v_{-j_{k}}=0$, which concludes the hypercyclicity of $T$.

Corollary 38. There exists an operator $T$ on a Banach space $X$ such that the whole space $X$ is a mean Li-Yorke set for $T$.

Remark 39. Since mean Li-Yorke chaos and DC2 are equivalent for dynamical systems on compact metric spaces, it follows from [23, Theorem 3.1] that the conclusion of the above corollary is not possible for such dynamical systems. 
Remark 40. It is possible to modify slightly the example in Theorem 37 in order to obtain $T$ such that every non-zero vector is absolutely mean semi-irregular for both $T$ and $T^{-1}$ (thus, both operators are generically mean Li-Yorke chaotic), but neither $T$ nor $T^{-1}$ are completely absolutely mean irregular. To do this, the only change would be to set $v_{m_{k}}=1, k \in \mathbb{Z}$. That is,

(a) $n_{0}=-1, m_{0}=1, n_{1}=4, m_{-1}=-4, v_{n_{0}}=1, v_{m_{0}}=1, v_{n_{1}}=2^{-1 / 3}, v_{m_{-1}}=1$, and

(b) $m_{k}=-n_{-k}, k \in \mathbb{Z}, v_{n_{k}}=(2 k)^{-1 / 3}, v_{m_{k}}=1, v_{n_{-k}}=(2 k+1)^{-1 / 3}, v_{m_{-k}}=1, k \in \mathbb{N}$, $v_{i} / v_{i-1}=v_{j} / v_{j-1}$ if $\left.\left.i, j \in\right] n_{k}, m_{k}\right]$, or if $\left.\left.i, j \in\right] m_{k-1}, n_{k}\right], k \in \mathbb{Z}$, and

(c) $m_{k}=\left(16 k^{3}+1\right) n_{k}, n_{k+1}=\left(16 k^{3}+1\right) m_{k}, k \in \mathbb{N}$.

In that case, the vectors of the unit basis are not absolutely mean irregular. Also, we observe that the hypercyclicity condition is preserved.

\section{Mean Li-Yorke chaotic semigroups}

We recall that a one-parameter family $\left(T_{t}\right)_{t>0}$ of operators on $X$ is called a $C_{0}$ semigroup if $T_{0}=I, T_{t} T_{s}=T_{t+s}(t, s \geq 0)$ and $\lim _{t \rightarrow s} T_{t} x=T_{s} x(x \in X$ and $s \geq 0)$. It is well-known that such a semigroup is always locally equicontinuous, in the sense that

$$
\sup _{t \in[0, b]}\left\|T_{t}\right\|<\infty \text { for every } b>0
$$

We refer the reader to the book [21] for a detailed study of $C_{0^{-}}$-semigroups. In the sequel, $\mathcal{T}=\left(T_{t}\right)_{t \geq 0}$ will denote an arbitrary $C_{0}$-semigroup, unless otherwise specified.

Definition 41. $\mathcal{T}$ is said to be mean Li-Yorke chaotic if there is an uncountable subset $S$ of $X$ (a mean Li-Yorke set for $\mathcal{T}$ ) such that every pair $(x, y)$ of distinct points in $S$ is a mean $L i$-Yorke pair for $\mathcal{T}$, in the sense that

$$
\liminf _{b \rightarrow \infty} \frac{1}{b} \int_{0}^{b}\left\|T_{t} x-T_{t} y\right\| d t=0 \quad \text { and } \quad \limsup _{b \rightarrow \infty} \frac{1}{b} \int_{0}^{b}\left\|T_{t} x-T_{t} y\right\| d t>0 .
$$

If $S$ can be chosen to be dense (resp. residual) in $X$, then we say that $\mathcal{T}$ is densely (resp. generically) mean Li-Yorke chaotic.

Li-Yorke chaos and distributional chaos for $C_{0}$-semigroups were studied in $[1,2,3,17$, 19, 37], for instance.

Definition 42. $\mathcal{T}$ is called absolutely Cesàro bounded if there is $C>0$ such that

$$
\sup _{b>0} \frac{1}{b} \int_{0}^{b}\left\|T_{t} x\right\| d t \leq C\|x\| \text { for all } x \in X .
$$

Definition 43. We say that $x \in X$ is an absolutely mean irregular (resp. absolutely mean semi-irregular) vector for $\mathcal{T}$ if

$$
\liminf _{b \rightarrow \infty} \frac{1}{b} \int_{0}^{b}\left\|T_{t} x\right\| d t=0 \quad \text { and } \quad \limsup _{b \rightarrow \infty} \frac{1}{b} \int_{0}^{b}\left\|T_{t} x\right\| d t=\infty(\text { resp. }>0) .
$$


Definition 44. An absolutely mean irregular manifold for $\mathcal{T}$ is a vector subspace $Y$ of $X$ such that every nonzero vector in $Y$ is absolutely mean irregular for $\mathcal{T}$.

As we did in [18] for hypercyclicity, or in [1] for distributional chaos, we will establish equivalences between the above notions for $C_{0}$-semigroups and the corresponding ones for the operators of the semigroup. The following simple lemma (whose proof we omit) will be very useful for this purpose.

Lemma 45. For each $s>0$, let $C_{s}:=\sup _{t \in[0, s]}\left\|T_{t}\right\|<\infty$. Then

$$
\frac{1}{C_{s}} \frac{1}{N+1} \sum_{j=1}^{N}\left\|\left(T_{s}\right)^{j} x\right\| \leq \frac{1}{b} \int_{0}^{b}\left\|T_{t} x\right\| d t \leq C_{s} \frac{1}{N} \sum_{j=0}^{N}\left\|\left(T_{s}\right)^{j} x\right\|,
$$

whenever $x \in X$ and $N s \leq b<(N+1) s$ with $N \geq 1$.

The next two propositions follow easily from this lemma.

Proposition 46. Given $x \in X$ and a $C_{0}$-semigroup $\mathcal{T}$, the following are equivalent:

1. $x$ is an absolutely mean semi-irregular (resp. irregular) vector for $\mathcal{T}$;

2. there exists $s>0$ such that $x$ is an absolutely mean semi-irregular (resp. irregular) vector for $T_{s}$;

3. $x$ is an absolutely mean semi-irregular (resp. irregular) vector for $T_{s}$, for all $s>0$.

Proposition 47. Given a $C_{0}$-semigroup $\mathcal{T}$, the following are equivalent:

1. $\mathcal{T}$ is absolutely Cesàro bounded (resp. (densely, generically) mean Li-Yorke chaotic, admits a dense absolutely mean irregular manifold);

2. there exists $s>0$ such that $T_{s}$ is absolutely Cesàro bounded (resp. (densely, generically) mean Li-Yorke chaotic, admits a dense absolutely mean irregular manifold);

3. $T_{s}$ is absolutely Cesàro bounded (resp. (densely, generically) mean Li-Yorke chaotic, admits a dense absolutely mean irregular manifold), for all $s>0$.

Remark 48. With these propositions at hand, it is easy to transport many of our previous theorems on operators to the semigroup setting. For instance, Theorems 5 (without (v)), 17 and 33 remain valid if we replace the operator $T$ by the semigroup $\mathcal{T}$. In particular, absolutely Cesàro bounded $C_{0}$-semigroups are never mean Li-Yorke chaotic.

Theorem 49. Suppose that the set $\left\{x \in X: \lim _{b \rightarrow \infty} \frac{1}{b} \int_{0}^{b}\left\|T_{t} x\right\| d t=0\right\}$ is dense in $X$. If any of the following conditions is true:

(a) $\limsup _{b \rightarrow \infty} \frac{1}{b} \int_{0}^{b}\left\|T_{t} y_{0}\right\| d t>0$ for some $y_{0} \in X$,

(b) $X$ is a Banach space and $\limsup _{t \rightarrow \infty} \frac{\left\|T_{t}\right\|}{t}>0$,

(c) $X$ is a Hilbert space and $\limsup _{t \rightarrow \infty} \frac{\left\|T_{t}\right\|}{t^{\frac{1}{2}}}>0$, 
(d) there is some $\lambda \in \sigma_{p}(A)$ with $\operatorname{Re} \lambda \geq 0$, where $A$ is the infinitesimal generator of $\mathcal{T}$, then $\mathcal{T}$ has a residual set of absolutely mean irregular vectors. If, in addition, $X$ is separable, then $\mathcal{T}$ admits a dense absolutely mean irregular manifold.

Proof. It is a consequence of Theorem 22 and Corollary 25 (note that (b) and (c) imply that $\limsup _{n \rightarrow \infty} \frac{\left\|T_{1}^{n}\right\|}{n}>0$ and $\limsup _{n \rightarrow \infty} \frac{\left\|T_{1}^{n}\right\|}{n^{\frac{1}{2}}}>0$, respectively). For the last assertion, see Remark 32 .

As an immediate consequence of the previous theorem, we have the following dichotomy for translation semigroups on weighted $L^{p}$ spaces.

Corollary 50. Let $v: \mathbb{R}_{+} \rightarrow \mathbb{R}$ be an admissible weight function and consider the translation semigroup $\mathcal{T}$, given by

$$
T_{t}(f)(x)=f(x+t), \quad t, x \geq 0,
$$

on the space $L_{v}^{p}\left(\mathbb{R}_{+}\right)[26$, Example 7.4]. Then either

(a) $\lim _{b \rightarrow \infty} \frac{1}{b} \int_{0}^{b}\left\|T_{t} f\right\| d t=0$ for every $f \in L_{v}^{p}\left(\mathbb{R}_{+}\right)$, or

(b) $\mathcal{T}$ admits a dense absolutely mean irregular manifold.

As a consequence of Theorem 49(b) and [35, page 224], we obtain

Corollary 51. The $C_{0}$-semigroup $\mathcal{T}$ defined on $L^{1}(1, \infty)$ by

$$
T_{t} f(x):=\left(\frac{x+t}{x}\right) f(x+t)
$$

is mean Li-Yorke chaotic.

Question 52. Is there a $C_{0}$-semigroup which is mean Li-Yorke chaotic but is not distributionally chaotic?

Theorem 53. There exists a mixing absolutely Cesàro bounded $C_{0}$-semigroup $\mathcal{T}$ on $L^{p}(1, \infty)$ for $1 \leq p<\infty$.

Proof. Let $0<\varepsilon<1$ and consider the weighted translation semigroup $\left(T_{t}\right)$ on $L^{p}(1, \infty)$ defined by

$$
T_{t} f(x):=\left(\frac{x+t}{x}\right)^{\frac{1-\varepsilon}{p}} f(x+t) .
$$


Given $f \in L^{p}(1, \infty)$ with $\|f\|_{p}=1$, and $b>0$, we have that

$$
\begin{aligned}
\int_{0}^{b}\left\|T_{t} f\right\|^{p} d t & =\int_{0}^{b}\left(\int_{1}^{\infty}\left(\frac{x+t}{x}\right)^{1-\varepsilon}|f(x+t)|^{p} d x\right) d t \\
& =\int_{0}^{b}\left(\int_{1+t}^{\infty}\left(\frac{x}{x-t}\right)^{1-\varepsilon}|f(x)|^{p} d x\right) d t \\
& =\int_{1}^{\infty} x^{1-\varepsilon}|f(x)|^{p} \int_{0}^{\min \{x-1, b\}}\left(\frac{1}{x-t}\right)^{1-\varepsilon} d t d x \\
& \leq \int_{1}^{1+2 b} x^{1-\varepsilon}|f(x)|^{p} \int_{0}^{x-1}\left(\frac{1}{x-t}\right)^{1-\varepsilon} d t d x \\
& +\int_{1+2 b}^{\infty}|f(x)|^{p} \int_{0}^{b}\left(\frac{x}{x-t}\right)^{1-\varepsilon} d t d x \\
\leq & \int_{1}^{1+2 b} \frac{x-1}{\varepsilon}|f(x)|^{p} d x+2 b \leq\left(2+\frac{2}{\varepsilon}\right) b .
\end{aligned}
$$

So,

$$
\left(\frac{1}{b} \int_{0}^{b}\left\|T_{t} f\right\| d t\right)^{p} \leq \frac{1}{b} \int_{0}^{b}\left\|T_{t} f\right\|^{p} d t \leq 2+\frac{2}{\varepsilon} .
$$

Thus, $\left(T_{t}\right)$ is an absolutely Cesàro bounded $C_{0}$-semigroup. Let us now see that $\left(T_{t}\right)$ is mixing. If $v(x):=\left(\frac{1}{x}\right)^{1-\varepsilon}$, then $T_{t}$ can be rewritten as

$$
T_{t} f(x)=\left(\frac{v(x)}{v(x+t)}\right)^{\frac{1}{p}} f(x+t) .
$$

Since $v$ is an admissible weight function, the translation semigroup defined as

$$
\tau_{t} f(x):=f(x+t)
$$

is a $C_{0}$-semigroup on $L_{v}^{p}(1, \infty)$. Moreover, $T_{t}$ on $L^{p}(1, \infty)$ and $\tau_{t}$ on $L_{v}^{p}(1, \infty)$ are topologically conjugate. Since $\left(\tau_{t}\right)$ is mixing because $\lim _{x \rightarrow \infty} v(x)=0$ [26, Example 7.10(b)], we conclude that $\left(T_{t}\right)$ is also mixing.

Corollary 54. There exists a mixing not absolutely mean irregular $C_{0}$-semigroup $\mathcal{T}$ on $L^{p}(1, \infty)$ for $1 \leq p<\infty$.

Theorem 55. There exists a forward translation $C_{0}$-semigroup $\mathcal{T}$ on $L_{v}^{p}\left(\mathbb{R}_{+}\right)$which is distributionally chaotic and not mean Li-Yorke chaotic.

Proof. It was proved in [13, Theorem 25] that there is a sequence $w=\left(w_{n}\right)_{n \in \mathbb{N}}$ of positive weights such that the unilateral weighted forward shift

$$
F_{w}:\left(x_{1}, x_{2}, \ldots\right) \in \ell^{p}(\mathbb{N}) \mapsto\left(0, w_{1} x_{1}, w_{2} x_{2}, \ldots\right) \in \ell^{p}(\mathbb{N})
$$

is distributionally chaotic and satisfies

$$
\lim _{N \rightarrow \infty} \frac{1}{N} \sum_{j=1}^{N}\left\|\left(F_{w}\right)^{j} x\right\|=\infty \quad \text { for all } x \in \ell^{p}(\mathbb{N}) \backslash\{0\} .
$$

By using conjugacy, we see that there is a sequence $v^{\prime}=\left(v_{n}\right)_{n \in \mathbb{N}}$ of positive weights such that the unweighted forward shift on $\ell^{p}\left(v^{\prime}, \mathbb{N}\right)$ is distributionally chaotic and not mean LiYorke chaotic. Now, if we consider as admissible weight function $v$ the polygonal formed by the sequence $v^{\prime}$, then the forward translation $C_{0}$-semigroup on $L_{v}^{p}\left(\mathbb{R}_{+}\right)$is distributionally chaotic and not mean Li-Yorke chaotic. 
Remark 56. The example in Theorem 37 can be easily adapted to the semigroup setting in order to construct a completely absolutely mean irregular $C_{0}$-semigroup $\mathcal{T}$. Indeed, the translation semigroup on $L_{v}^{p}(\mathbb{R})$ does the job if we fix $v(k)=v_{k}, k \in \mathbb{Z}$, where $\left(v_{k}\right)_{k \in \mathbb{Z}}$ is the sequence of weights of the example in Theorem 37, and we set $v(x)=v(k)$, $x \in] k-1, k], k \in \mathbb{Z}$.

\section{Acknowledgements}

This work was partially done on a visit of the first author to the Institut Universitari de Matemàtica Pura i Aplicada at Universitat Politècnica de València, and he is very grateful for the hospitality and support. The first author was partially supported by CNPq and by grant \#2017/22588-0, São Paulo Research Foundation (FAPESP). The second and third authors were supported by MINECO and FEDER, Project MTM2016-75963-P. The third author was also supported by Generalitat Valenciana, Project PROMETEO/2017/102. We thank Frédéric Bayart for providing us Theorem 27, which answers a previous question of us. We also thank the referee whose careful comments produced an improvement in the presentation of the article.

\section{References}

[1] A. A. Albanese, X. Barrachina, E. M. Mangino and A. Peris, Distributional chaos for strongly continuous semigroups of operators, Commun. Pure Appl. Anal. 12 (2013), no. 5, 2069-2082.

[2] X. Barrachina and J. A. Conejero, Devaney chaos and distributional chaos in the solution of certain partial differential equations, Abstr. Appl. Anal. 2012, Art. ID 457019, 11 pp.

[3] X. Barrachina and A. Peris, Distributionally chaotic translation semigroups, J. Difference Equ. Appl. 18 (2012), no. 4, 751-761.

[4] F. Bayart and S. Grivaux, Frequently hypercyclic operators, Trans. Amer. Math. Soc. 358 (2006), no. 11, 5083-5117.

[5] F. Bayart and É. Matheron, Dynamics of Linear Operators, Cambridge University Press, Cambridge, 2009.

[6] F. Bayart, F. and I. Z. Ruzsa, Difference sets and frequently hypercyclic weighted shifts, Ergodic Theory Dynam. Systems 35 (2015), no. 3, 691-709.

[7] M. J. Beltrán-Meneu, Operators on weighted spaces of holomorphic functions, PhD Thesis, Universitat Politècnica de València, 2014.

[8] T. Bermúdez, A. Bonilla, F. Martínez-Giménez and A. Peris, Li-Yorke and distributionally chaotic operators, J. Math. Anal. Appl. 373 (2011), no. 1, 83-93.

[9] T. Bermúdez, A. Bonilla, V. Müller and A. Peris, Cesàro bounded operators in Banach spaces, J. Anal. Math. (to appear). 
[10] L. Bernal-González and A. Bonilla, Order of growth of distributionally irregular entire functions for the differentiation operator, Complex Var. Elliptic Equ. 61 (2016), no. 8, 1176-1186.

[11] N. C. Bernardes Jr., A. Bonilla, V. Müller and A. Peris, Distributional chaos for linear operators, J. Funct. Anal. 265 (2013), no. 9, 2143-2163.

[12] N. C. Bernardes Jr., A. Bonilla, V. Müller and A. Peris, Li-Yorke chaos in linear dynamics, Ergodic Theory Dynam. Systems 35 (2015), no. 6, 1723-1745.

[13] N. C. Bernardes Jr., A. Bonilla, A. Peris and X. Wu, Distributional chaos for operators on Banach spaces, J. Math. Anal. Appl. 459 (2018), no. 2, 797-821.

[14] N. C. Bernardes Jr., A. Peris and F. Rodenas, Set-valued chaos in linear dynamics, Integral Equations Operator Theory 88 (2017), no. 4, 451-463.

[15] J. Bès, Q. Menet, A. Peris and Y. Puig, Recurrence properties of hypercyclic operators, Math. Ann. 366 (2016), no. 1-2, 545-572.

[16] A. Bonilla and K.-G. Grosse-Erdmann, Frequently hypercyclic operators and vectors, Ergodic Theory Dynam. Systems 27 (2007), no. 2, 383-404. Erratum: Ergodic Theory Dynam. Systems 29 (2009), no. 6, 1993-1994.

[17] J. A. Conejero, C. Lizama, M. Murillo-Arcila and A. Peris, Linear dynamics of semigroups generated by differential operators, Open Math. 15 (2017), 745-767.

[18] J. A. Conejero, V. Müller and A. Peris, Hypercyclic behaviour of operators in a hypercyclic $C_{0}$-semigroup, J. Funct. Anal. 244 (2007), no. 1, 342-348.

[19] J. A. Conejero, F. Rodenas and M. Trujillo, Chaos for the hyperbolic bioheat equation, Discrete Contin. Dyn. Syst. 35 (2015), no. 2, 653-668.

[20] T. Downarowicz, Positive topological entropy implies chaos DC2, Proc. Amer. Math. Soc. 142 (2014), no. 1, 137-149.

[21] K.-J. Engel and R. Nagel, One-Parameter Semigroups for Linear Evolution Equations, Springer-Verlag, New York - Berlin, 2000.

[22] N. S. Feldman, Hypercyclicity and supercyclicity for invertible bilateral weighted shifts, Proc. Amer. Math. Soc. 131 (2003), no. 2, 479-485.

[23] M. Foryś-Krawiec, P. Oprocha and M. Štefánková, Distributionally chaotic systems of type 2 and rigidity, J. Math. Anal. Appl. 452 (2017), no. 1, 659-672.

[24] F. Garcia-Ramos and L. Jin, Mean proximality and mean Li-Yorke chaos, Proc. Amer. Math. Soc. 145 (2017), no. 7, 2959-2969.

[25] S. Grivaux and É. Matheron, Invariant measures for frequently hypercyclic operators, Adv. Math. 265 (2014), 371-427.

[26] K.-G. Grosse-Erdmann and A. Peris Manguillot, Linear Chaos, Springer, London, 2011. 
[27] B. Hou, P. Cui and Y. Cao, Chaos for Cowen-Douglas operators, Proc. Amer. Math. Soc. 138 (2010), no. 3, 929-936.

[28] B. Hou and L. Luo, Some remarks on distributional chaos for bounded linear operators, Turkish J. Math. 39 (2015), no. 2, 251-258.

[29] W. Huang, J. Li and X. Ye, Stable sets and mean Li-Yorke chaos in positive entropy systems, J. Funct. Anal. 266 (2014), no. 6, 3377-3394.

[30] F. León-Saavedra, Operators with hypercyclic Cesàro means, Studia Math. 152 (2002), no. 3, 201-215.

[31] J. Li, S. Tu and X. Ye, Mean equicontinuity and mean sensitivity, Ergodic Theory Dynam. Systems 35 (2015), no. 8, 2587-2612.

[32] F. Martínez-Giménez, P. Oprocha and A. Peris, Distributional chaos for backward shifts, J. Math. Anal. Appl. 351 (2009), no. 2, 607-615.

[33] F. Martínez-Giménez, P. Oprocha and A. Peris, Distributional chaos for operators with full scrambled sets, Math. Z. 274 (2013), no. 1-2, 603-612.

[34] Q. Menet, Linear chaos and frequent hypercyclicity, Trans. Amer. Math. Soc. 369 (2017), no. 7, 4977-4994.

[35] V. Müller and J. Vršovský, Orbits of linear operators tending to infinity, Rocky Mountain J. Math. 39 (2009), no. 1, 219-230.

[36] J. Smítal and M. Štefánková, Distributional chaos for triangular maps, Chaos Solitons Fractals 21 (2004), no. 5, 1125-1128.

[37] X. Wu, Li-Yorke chaos of translation semigroups, J. Difference Equ. Appl. 20 (2014), no. $1,49-57$.

[38] X. Wu, L. Wang and G. Chen, Weighted backward shift operators with invariant distributionally scrambled subsets, Ann. Funct. Anal. 8 (2017), no. 2, 199-210.

[39] X. Wu, P. Oprocha and G. Chen, On various definitions of shadowing with average error in tracing, Nonlinearity 29 (2016), no. 7, 1942-1972.

[40] Z. Yin and Q. Yang, Generic distributional chaos and principal measure in linear dynamics, Ann. Polon. Math. 118 (2016), no. 1, 71-94.

[41] Z. Yin and Q. Yang, Distributionally n-scrambled set for weighted shift operators, J. Dyn. Control Syst. 23 (2017), no. 4, 693-708.

[42] Z. Yin and Q. Yang, Distributionally n-chaotic dynamics for linear operators, Rev. Mat. Complut. 31 (2018), no. 1, 111-129.

N. C. Bernardes Jr.

Departamento de Matemática Aplicada, Instituto de Matemática, Universidade Federal do Rio de Janeiro, Caixa Postal 68530, Rio de Janeiro, RJ 21945-970, Brazil.

e-mail: ncbernardesjr@gmail.com

A. Bonilla

Departamento de Análisis Matemático, Universidad de la Laguna, 38271, La Laguna (Tenerife), 
Spain.

e-mail: abonilla@ull.es

A. Peris

Institut Universitari de Matemàtica Pura i Aplicada, Universitat Politècnica de València, Edifici 8E, Acces F, 4a planta, 46022 València, Spain.

e-mail: aperis@mat.upv.es 